\title{
On longevity of I-ball/oscillon
}

\author{
Kyohei Mukaida, ${ }^{a}$ Masahiro Takimoto ${ }^{b, c}$ and Masaki Yamada ${ }^{d, e}$ \\ ${ }^{a}$ Kavli IPMU (WPI), UTIAS, The University of Tokyo, \\ Kashiwa, Chiba 277-8583, Japan \\ ${ }^{b}$ Theory Center, KEK, \\ 1-1 Oho, Tsukuba, Ibaraki 305-0801, Japan \\ ${ }^{c}$ Department of Particle Physics and Astrophysics, Weizmann Institute of Science, \\ Rehovot 7610001, Israel \\ ${ }^{d}$ Institute of Cosmology, Department of Physics and Astronomy, Tufts University, \\ Medford, MA 02155, U.S.A. \\ e Department of Physics, Tohoku University, \\ Sendai, Miyagi 980-8578, Japan \\ E-mail: kyohei.mukaida@ipmu.jp, masahiro.takimoto@weizmann.ac.il, \\ Masaki.Yamada@tufts.edu
}

ABSTRACT: We study I-balls/oscillons, which are long-lived, quasi-periodic, and spatially localized solutions in real scalar field theories. Contrary to the case of Q-balls, there is no evident conserved charge that stabilizes the localized configuration. Nevertheless, in many classical numerical simulations, it has been shown that they are extremely longlived. In this paper, we clarify the reason for the longevity, and show how the exponential separation of time scales emerges dynamically. Those solutions are time-periodic with a typical frequency of a mass scale of a scalar field. This observation implies that they can be understood by the effective theory after integrating out relativistic modes. We find that the resulting effective theory has an approximate global U(1) symmetry reflecting an approximate number conservation in the non-relativistic regime. As a result, the profile of those solutions is obtained via the bounce method, just like Q-balls, as long as the breaking of the $\mathrm{U}(1)$ symmetry is small enough. We then discuss the decay processes of the I-ball/oscillon by the breaking of the U(1) symmetry, namely the production of relativistic modes via number violating processes. We show that the imaginary part is exponentially suppressed, which explains the extraordinary longevity of I-ball/oscillon. In addition, we find that there are some attractor behaviors during the evolution of I-ball/oscillon that further enhance the lifetime. The validity of our effective theory is confirmed by classical numerical simulations. Our formalism may also be useful to study condensates of ultra light bosonic dark matter, such as fuzzy dark matter, and axion stars, for instance.

KEYwords: Beyond Standard Model, Cosmology of Theories beyond the SM

ArXiv EPRINT: 1612.07750 


\section{Contents}

1 Introduction 1

2 I-ball/oscillon as non-relativistic condensates 3

2.1 Non-relativistic effective field theory 3

2.2 I-ball/oscillon as a pseudo nontopological soliton 8

$\begin{array}{ll}2.3 \text { Decay of I-ball/oscillon via U(1) breaking } & 11\end{array}$

3 Numerical simulation $\quad \mathbf{1 5}$

$\begin{array}{lll}3.1 & \text { Case with } \mathbb{Z}_{2} \text { symmetry } & 17\end{array}$

$\begin{array}{lll}3.2 & \text { Case without } \mathbb{Z}_{2} \text { symmetry } & 19\end{array}$

$\begin{array}{ll}3.3 & \text { Case of double well potential }\end{array}$

4 Conclusions and discussion $\quad 22$

$\begin{array}{ll}\text { A Interaction terms } & 23\end{array}$

$\begin{array}{ll}\text { B Higher order terms } & 25\end{array}$

\section{Introduction}

Condensates of scalar fields play important roles in the early Universe. One of the most prominent examples is the inflaton field which causes the accelerated expansion of the Universe, i.e., inflation [1,2], and may seed primordial density fluctuations [3]. A curvaton field [4-6] is another candidate to generate the primordial density fluctuations. As the Higgs field in the Standard Model, some scalar field may realize a phase transition that leads to a spontaneous symmetry breaking (SSB). One of the most important examples of this kind is the SSB of Peccei-Quinn (PQ) symmetry, which is introduced to explain the strong CP problem [7]. The SSB results in a prediction of a pseudo-Nambu-Goldstone boson called axion [8] and it is known that the sizable amount of axion can be produced in the form of condensate by the misalignment mechanism [9-11]. In the Affleck-Dine baryogenesis scenario [12-14], baryonic $\mathrm{U}(1)$ charged scalar condensates are indispensable to generate the baryon asymmetry of the Universe.

Some scalar fields come to form (quasi-)stable and localized objects in the early stage of the Universe. Since the formation and time evolution of such localized objects may significantly affect the cosmological scenarios, it is important to understand their dynamics. In general, their existence is ensured by some conserved quantities. For example, the conservation of topological numbers will lead to the formation of monopoles, cosmic strings or domain walls, that are called as topological defects [15]. Such objects are particularly important to consider axion cosmology because cosmic strings form at the SSB 
of PQ symmetry and domain walls may form at the QCD phase transition depending on the model $[16,17]$. Another example of localized objects is so-called Q-balls [18-24] which appear in complex scalar theories and whose existence is ensured by the global U(1) symmetry. In any case, the conserved quantity seems to be the fundamental essence of their stability.

I-balls/oscillons [25-36, 36-47] are long-lived (but not absolutely stable), quasiperiodic, and spatially localized objects which appear in real scalar field theories if the potential is shallower than quadratic. The existence of such objects seems to be mysterious because there are no evident conserved quantities related to them. Nevertheless, many classical numerical studies have revealed that they are extremely long-lived. In ref. [31], it was discussed that the existence of I-balls/oscillons may be related to the (approximate) conservation of the adiabatic charge $I$ in the non-relativistic regime. In ref. [46], two of the authors found that the existence of I-ball/oscillon may be understood by the approximate number conservation in non-relativistic regime [48-50]. Those observations suggest that an approximately conserved quantity (number or adiabatic charge) in the non-relativistic regime of real scalar theories may play a major role in the existence and longevity of I-balls/oscillons.

The main purpose of this paper is to clarify the reason for the longevity of I-balls/oscillons, revealed in many classical numerical simulations, from the viewpoint in [46] (See refs. [26, 36-38] for different approaches). To do so, we construct an effective theory of real scalar field theories with polynomial potentials suitable to describe the dynamics in the non-relativistic regime. By integrating out relativistic modes, we obtain a non-relativistic effective theory. The resulting effective theory comes to have an approximate global U(1) symmetry, which reflects the approximate number conservation in the non-relativistic regime (See ref. [51] for the same viewpoint in turbulence). In this approach, I-ball/oscillon can be understood by the same way as that of Q-ball, as long as the breaking of the $\mathrm{U}(1)$ symmetry is small enough. This breaking is imprinted in the imaginary part of the effective theory, which reflects the production of relativistic particles via number violating processes. By omitting all the loop diagrams in the effective action, we explicitly show the smallness of the imaginary part. This explains the reason why the classical decay rate is so suppressed. (See refs. [52-54] and section 4 for quantum decays.) In particular, we find that there are some approximate attractor behaviors during the evolution of I-ball/oscillon. As a result, the lifetime of I-ball/oscillon is enhanced by many orders of magnitude. We also confirm the validity of our description by comparing the results of our effective theory with the numerical simulations of the original relativistic scalar field theory.

This paper is organized as follows. In section 2, we first explain how to construct the effective theory of the scalar field in the non-relativistic regime. Then, we demonstrate how to obtain the profile of I-balls/oscillons in the same way as Q-balls, and why they are so long-lived from the viewpoint of the approximate conservation of the U(1) symmetry. In section 3, we compare the results of our effective theory obtained in section 2 with those of numerical simulations of the original relativistic scalar field theory. Section 4 is devoted to conclusions and discussion. Possible impacts of quantum decays are briefly mentioned. 


\section{I-ball/oscillon as non-relativistic condensates}

In this section, we will argue that I-balls/oscillons can be regarded as pseudo nontopological solitons in the non-relativistic limit of real scalar field theories. This is because an approximate $\mathrm{U}(1)$ symmetry associated with the number conservation emerges in the nonrelativistic regime. Since this symmetry is not exact, those pseudo nontopological solitons eventually decay, whose time scale is controlled by the breaking of this symmetry.

First, we will sketch the way how to derive a non-relativistic effective theory from a relativistic real scalar. After integrating out relativistic modes, we will find that the approximate $\mathrm{U}(1)$ symmetry appears with respect to the number conservation. The breaking of $U(1)$ symmetry, which is inevitable because the interacting relativistic real scalar field does not conserve its number, can be identified as the imaginary part of the effective action. The breaking corresponds to the production of relativistic modes.

Next, we discuss the property of I-balls/oscillons, treating the imaginary part perturbatively. Namely, we first find a non-trivial spatially localized solution, i.e., I-ball/oscillon, neglecting the imaginary part, and then discuss its decay taking account of the imaginary part at the first order. If the decay time is much longer than the frequency of these solutions, we can regard them as pseudo nontopological solitons associated with the approximate $\mathrm{U}(1)$ symmetry. Interestingly, the properties of I-balls/oscillons strongly depend on whether or not the scalar potential respects a $\mathbb{Z}_{2}$ symmetry at the vacuum, $(\phi-\langle\phi\rangle) \mapsto-(\phi-\langle\phi\rangle){ }^{1}$

Finally, we discuss the classical decay of I-ball/oscillon via the breaking of U(1) symmetry by employing a simple model. We explicitly show the smallness of the imaginary part, which indicates the reason why the I-balls/oscillons are so long-lived in many classical numerical simulations. We also find several critical values of the charge $Q$ where the dominant decay channel is suppressed. As a result, I-balls/oscillons stay at such critical points in most of their lifetime. Those results are confirmed by numerical simulations of the original relativistic scalar field theory in section 3.

\subsection{Non-relativistic effective field theory}

Let us start with the following relativistic real scalar field theory:

$$
\mathcal{L}=\frac{1}{2} \partial_{\mu} \phi \partial^{\mu} \phi-\frac{1}{2} m_{\phi}^{2} \phi^{2}-V_{\text {int }}(\phi)
$$

where $\phi$ is a real scalar field, $m_{\phi}$ is its mass, and $V_{\text {int }}$ is the interaction term. The goal of this subsection is to derive an effective action which describes the non-relativistic motion of $\phi$ by integrating out relativistic modes from this Lagrangian.

We divide the scalar field $\phi$ into two parts; the non-relativistic part and the other:

$$
\phi(x)=\phi_{\mathrm{NR}}(x)+\delta \phi(x)
$$

\footnotetext{
${ }^{1}$ The potential with an explicit $\mathbb{Z}_{2}$ breaking term falls into this case. In addition, even if the potential respects the $\mathbb{Z}_{2}$ symmetry, the vacuum state can break it spontaneously. This case also falls into the $\mathbb{Z}_{2}$ breaking one in our classification.
} 
where

$$
\phi_{\mathrm{NR}}(x) \equiv \int_{K \in \mathrm{NR}} e^{-i K \cdot x} \phi(K), \quad \delta \phi(x) \equiv \int_{K \in \overline{\mathrm{NR}}} e^{-i K \cdot x} \phi(K),
$$

with $\left(K^{\mu}\right)=\left(k^{0}, \boldsymbol{k}\right) . \phi(K)$ is the Fourier coefficient of the real scalar field, which satisfies $\phi(K)=\phi^{*}(-K)$. The word "NR" indicates the region which is close to the on-shell pole of non-relativistic excitations, i.e., $\mathrm{NR} \equiv\left\{\left(k_{0}, \boldsymbol{k}\right) \mid \pm k_{0} \sim m_{\phi}+\mathcal{O}\left(m_{\phi} v^{2}\right), \pm \boldsymbol{k} \sim \mathcal{O}\left(m_{\phi} \boldsymbol{v}\right)\right\}$ with $v \equiv|\boldsymbol{v}| \ll 1$. $\overline{\mathrm{NR}}$ is defined as its complementary set. For later convenience, we further split the non-relativistic part into two parts:

$$
\phi_{\mathrm{NR}}(x)=\frac{1}{2}\left[\Psi(t, \boldsymbol{x}) e^{-i m_{\phi} t}+\text { H.c. }\right],
$$

where

$$
\Psi(t, \boldsymbol{x})=\int_{K \in \mathrm{NR}_{+}} e^{-i\left(k_{0}-m_{\phi}\right) t+i \boldsymbol{k} \cdot \boldsymbol{x}} \phi(K)
$$

Here $\mathrm{NR}_{+}$represents a non-relativistic region with positive energy $k_{0}>0$. By definition, $\Psi$ is a slowly varying field

$$
\left|\frac{\partial^{2} \Psi}{\partial\left(m_{\phi} t\right)^{2}}\right| \ll\left|\frac{\partial \Psi}{\partial\left(m_{\phi} t\right)}\right| \ll|\Psi|
$$

and also its spatial gradient is small

$$
\left|\frac{\nabla^{2} \Psi}{\Psi}\right| \ll m_{\phi}^{2}
$$

The initial condition of the original real scalar field theory is mapped by

$$
\left.\phi\right|_{\text {ini }} \simeq \Re\left[\left.\Psi\right|_{\text {ini }}\right],\left.\quad \dot{\phi}\right|_{\text {ini }} \simeq m_{\phi} \Im\left[\left.\Psi\right|_{\text {ini }}\right] .
$$

We can derive the non-relativistic effective field theory by integrating out $\delta \phi$. Throughout this paper, we focus on the classical longevity of I-ball/oscillon in order to clarify the reason why the I-balls/oscillons are so long-lived in many classical numerical studies. Thus, we omit the loop contributions from the effective action so that we can obtain the classical non-relativistic effective field theory. Possible quantum effects will be discussed in section 4 . The property of the effective field theory depends on whether or not it respects a $\mathbb{Z}_{2}$ symmetry at the vacuum, i.e., $(\phi-\langle\phi\rangle) \mapsto-(\phi-\langle\phi\rangle)$. We illustrate what we are going to work on by taking simple examples for each case.

With $\mathbb{Z}_{\mathbf{2}}$ symmetry. First, let us assume that the theory respects a $\mathbb{Z}_{2}$ symmetry at the vacuum: $(\phi-\langle\phi\rangle) \mapsto-(\phi-\langle\phi\rangle)$. Without loss of generality, we take $\langle\phi\rangle=0$. To be concrete, suppose that $V_{\text {int }}=-g_{4} \phi^{4} / 4+\cdots$ dominates the potential. Here note that higher order terms are implicit, which are required to stabilize the vacuum at $\langle\phi\rangle=0$. The 
relevant interaction terms are

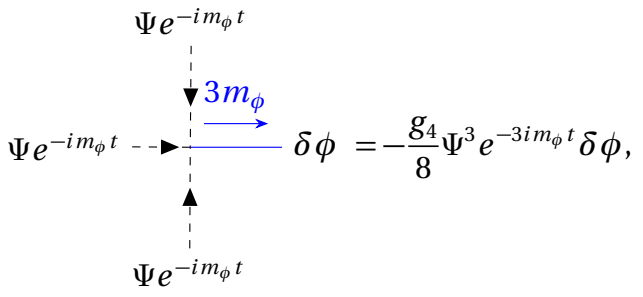<smiles>[Mg][Mg][Ba][Ba]</smiles>
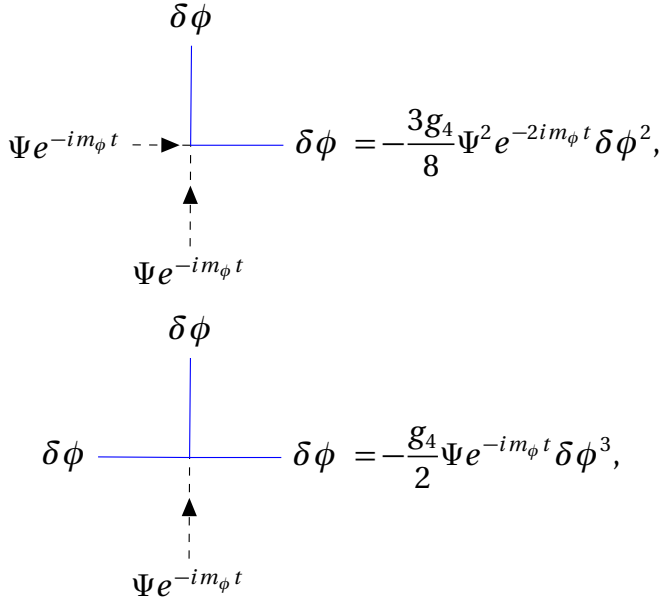

and those conjugates. ${ }^{2}$ Here a dashed line with an arrow represents a non-relativistic mode $\Psi$ or $\Psi^{\dagger}$, and a blue line is the other $\delta \phi$. Note that the terms, like $\delta \phi|\Psi|^{2 n}$, are forbidden by the energy conservation because the operator $|\Psi|^{2 n}$ cannot carry relativistic energies.

To obtain an effective theory which describes the dynamics of non-relativistic modes, we only keep $\Psi e^{-i m_{\phi} t}$ and $\Psi^{\dagger} e^{i m_{\phi} t}$ as external lines and integrate out $\delta \phi$. After integration, we obtain vertices such as $\Psi^{n} e^{-n i m_{\phi} t} \Psi^{\dagger^{m}} e^{\text {mim }_{\phi} t}$. Since the non-relativistic fields, $\Psi\left(\Psi^{\dagger}\right)$, cannot provide incoming (outgoing) energies comparable to the mass scale $m_{\phi}$, vertices except $n=m$ vanish owing to the energy conservation. As a result, all the interaction terms involve a pair of $\Psi \Psi^{\dagger}$, which respects the $\mathrm{U}(1)$ symmetry: $\Psi \mapsto e^{i \theta} \Psi$. This $\mathrm{U}(1)$ symmetry is nothing but the number conservation of non-relativistic particles.

To see how to integrate out $\delta \phi$, let us study the following diagram: ${ }^{3}$

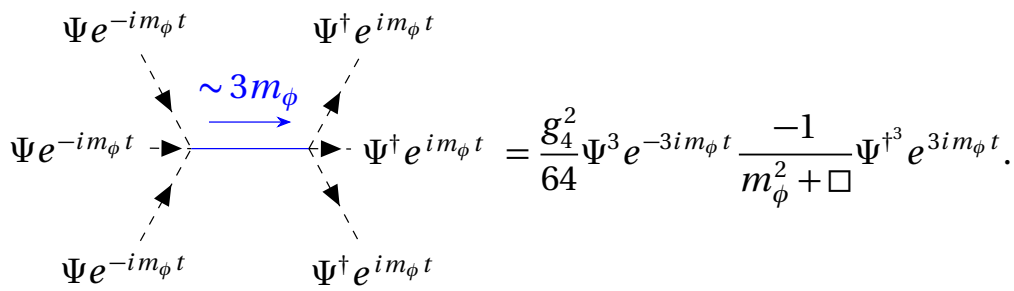

Let us first discard terms where $\partial_{\mu}$ acts on $\Psi$ because it is slowly varying and has small gradients. The leading order real part contribution comes from the term where $\partial_{t}^{2}$ acts on $e^{3 i m_{\phi} t}$. One finds the following corrections to the effective action, $\delta \mathcal{L} \supset-g_{4}^{2}|\Psi|^{6} /\left(512 m_{\phi}^{2}\right)+$ $\cdots$, where ellipses represent higher order contributions.

However, the number conservation is not exact for an interacting relativistic real scalar. The breaking of $\mathrm{U}(1)$ symmetry is imprinted in the imaginary part of the effective action after integration. Such an imaginary part comes from cuttings of diagrams which stand for

\footnotetext{
${ }^{2}$ The Hermite conjugate of $\left(3 g_{4} / 8\right)|\Psi|^{2} \delta \phi^{2}$ should also be included.

${ }^{3}$ Here we symbolically write the propagator as $-1 /\left(\square+m_{\phi}^{2}\right)$, but note that the $i \varepsilon$-term should be included properly, which reflects the physical boundary condition of this setup. See also section 2.3.
} 
the production of relativistic modes. To obtain the leading order imaginary part from the diagram of eq. (2.10), we have to keep terms where $\partial_{\mu}$ act on $\Psi$, contrary to the real part contributions. Although its fraction is tiny, $\Psi$ may contain higher momentum modes. If this is the case, the pole of eq. (2.10) can yield the imaginary part of the effective action, which indicates the production of relativistic modes with $k_{0} \sim 3 m_{\phi}, k \equiv|\boldsymbol{k}| \sim 2 \sqrt{2} m_{\phi}$.

In the following, we first neglect the imaginary part and find a spatially localized energetically favored state in section 2.2. Then, we discuss its decay. Treating the classical lump as a background, we will see that a tiny fraction of the obtained solution can hit the pole and produces relativistic modes in section 2.3.

Without $\mathbb{Z}_{2}$ symmetry. Then, let us add terms which break the $\mathbb{Z}_{2}$ symmetry at the vacuum: $(\phi-\langle\phi\rangle) \mapsto-(\phi-\langle\phi\rangle)$. Again we shift the field so that $\langle\phi\rangle=0$ without loss of generality. In this case, we have interaction terms, like $|\Psi|^{n} \delta \phi$, which do not contain a rapidly oscillating term, $e^{n i m_{\phi} t}$. The term, $\delta \phi(t, \boldsymbol{x})$, is not relativistic rather varying slowly. Nevertheless, it is far below the pole of non-relativistic excitations because its typical energy is $\left|k_{0}\right| \sim m_{\phi} v^{2} \ll m_{\phi}$. We denote such contributions as $\delta \phi_{V}$ so as to distinguish them from relativistic fluctuations in the following.

To be concrete, suppose that we have a cubic interaction, $g_{3} \phi^{3} / 3$. It yields the following interaction term involving $\delta \phi_{V}$ :

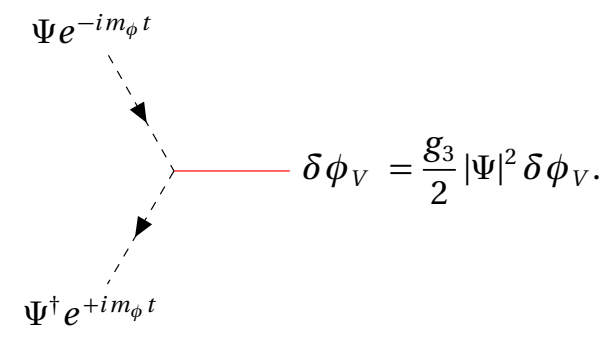

Here the red line is $\delta \phi_{V}$, which is slowly varying but far below the pole of non-relativistic excitations. Integrating out such fluctuations, one finds the following diagram

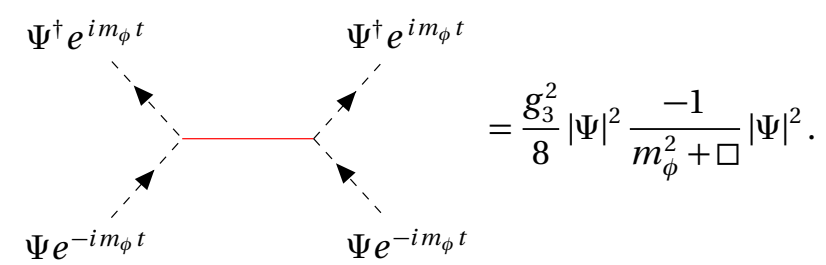

The additional $1 / 2$ comes from the symmetry factor of this diagram. Here, we can neglect the derivatives in the denominator in the leading approximation. As an another approach, it is instructive to see the equation of motion for $\delta \phi_{V}$. Suppose that the system somehow becomes (quasi-)static, which is the case for the I-ball and oscillon solution. Then, $\delta \phi_{V}$ should satisfy the following equation:

$$
\delta \phi_{V}=-\frac{g_{3}|\Psi|^{2}}{2 m_{\phi}^{2}}+\cdots .
$$


Substituting this into the Lagrangian, we obtain the same result as eq. (2.12). One can see that $\phi$ gets a constant shift due to the condensate of the non-relativistic field, $\Psi$. Note that its amplitude is smaller than $\Psi$, i.e., $\left|\delta \phi_{V}\right| \ll|\Psi|$, if the coupling is small.

We also have the following diagram involving an intermediate relativistic fluctuation,

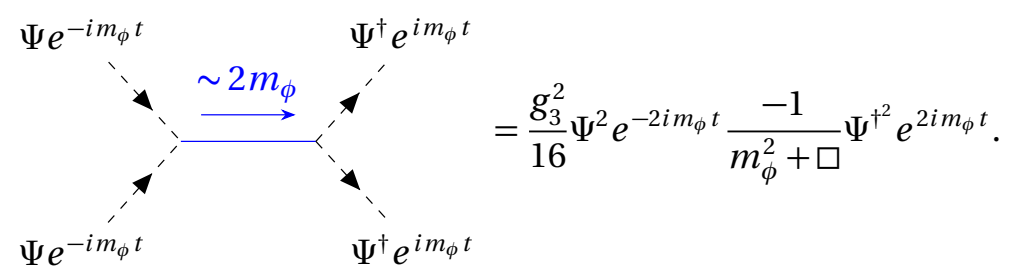

Again, one can obtain the leading correction to the real part of the effective action, omitting terms $\partial_{\mu}$ acting on $\Psi$. We find the following correction to the effective action: $\delta \mathcal{L} \supset$ $5 g_{3}^{2} /\left(48 m_{\phi}^{2}\right)|\Psi|^{4}+\cdots$. After integration, one can see that the vertices only involve pairs of $\Psi \Psi^{\dagger}$ owing to the energy conservation. Therefore, the real part of the effective action respects the $\mathrm{U}(1)$ symmetry: $\Psi \mapsto e^{i \theta} \Psi$.

On the other hand, the leading contribution to the imaginary part comes from the cutting of eq. (2.14). ${ }^{4}$ Plugging a spatially localized solution of $\Psi$ which is obtained by neglecting the imaginary part, we will see that the tiny fraction contains high momentum modes with $p \sim \sqrt{3} m_{\phi}$. Hence, the production of relativistic particles with $p_{0} \sim 2 m_{\phi}$ and $p \sim \sqrt{3} m_{\phi}$ takes place. See section 2.3 for details.

Summary. Here we summarize basic properties of the non-relativistic effective field theory which is obtained from integrating out $\delta \phi$ and also $\delta \phi_{V}$ from a relativistic real scalar field theory. As already explained, all the vertices appear with pairs of $\Psi \Psi^{\dagger}$ owing to the energy conservation, and hence the effective theory respects the $\mathrm{U}(1)$ symmetry: $\Psi \mapsto e^{i \theta} \Psi$. This symmetry corresponds to the number conservation of non-relativistic particles. Since an interacting relativistic scalar does not conserve the particle number, this symmetry should not be exact. The breaking of $\mathrm{U}(1)$ is imprinted in the imaginary part of the effective action, which stands for the production of relativistic modes.

To sum up, the effective theory for non-relativistic fields, $\Psi$, takes the form:

$$
S_{\mathrm{NR}}[\Psi]=\int_{x} \frac{1}{4}\left[\Psi^{\dagger}\left(2 i m_{\phi} \partial_{t}-\partial_{t}^{2}+\nabla^{2}\right) \Psi-V_{\mathrm{eff}}(|\Psi|)\right]-i \Gamma[\Psi] .
$$

We adopt the following potential as an example in the following discussion:

$$
V(\phi)=\frac{g_{3}}{3} \phi^{3}-\frac{g_{4}}{4} \phi^{4}+\frac{g_{6}}{6} \phi^{6} .
$$

By performing the same procedure demonstrated around eqs. (2.10), (2.12) and (2.14), one may obtain the effective potential perturbatively after some algebras:

$$
V_{\text {eff }}(|\Psi|)=\left(-\frac{5 g_{3}^{2}}{12 m_{\phi}^{2}}-\frac{3 g_{4}}{8}\right)|\Psi|^{4}+\left(\frac{5 g_{6}}{24}+\frac{g_{4}^{2}}{128 m_{\phi}^{2}}\right)|\Psi|^{6}+\cdots
$$

\footnotetext{
${ }^{4}$ It may be unlikely to hit the pole of eq. (2.12) because not only the spatial gradients but the incoming (outgoing) energy is expected to be much smaller than the mass scale $m_{\phi}$.
} 
Note that a factor of $-1 / 4$ should be multiplied to get the effective potential, $V_{\text {eff }}$, from the corrections to the Lagrangian $\delta \mathcal{L}$, since we have defined the effective action by eq. (2.15). We have omitted higher order terms; such as those with higher order in the coupling or those with $\partial_{\mu}$ acting on $\Psi$, so as to get the leading order corrections to the effective potential, for $\Psi$ varies slowly and has small spatial gradients. ${ }^{5}$ To discuss higher order terms in the coupling consistently, terms with $\partial_{\mu}$ acting on $\Psi$ should also be included. See appendix B for details.

The imaginary part of the effective action, $\Gamma[\Psi]$, may be dominated by the cutting diagrams of eqs. (2.10) or (2.14) for classical decays, as discussed in section 2.3.

\subsection{I-ball/oscillon as a pseudo nontopological soliton}

There exist two conserved quantities, its energy and charge associated with the U(1) symmetry, in the effective theory, if we neglect the production of relativistic modes induced via the imaginary part. The goal of this subsection is to derive a non-trivial spatially localized solution as an energetically favored state with a fixed charge. This treatment is justified a posteriori in section 2.3 , where we show that the decay rate induced by this imaginary part is much slower than the typical frequency of these solutions. Thus, at each time step, we can safely regard its energy and charge as conserved quantities and discuss its decay as an adiabatic process.

Conserved quantities. The $\mathrm{U}(1)$ charge associated with $\Psi \mapsto e^{i \theta} \Psi$ is given by

$$
Q=\frac{1}{4} i \int_{x}\left[\Psi^{\dagger}\left(\partial_{t} \Psi-i m_{\phi} \Psi\right)-\Psi\left(\partial_{t} \Psi^{\dagger}+i m_{\phi} \Psi^{\dagger}\right)\right]
$$

where $\int_{\boldsymbol{x}} \equiv \int \mathrm{d}^{3} \boldsymbol{x}$. And the energy is

$$
E=\frac{1}{4} \int_{\boldsymbol{x}}\left[\left|\partial_{t} \Psi-i m_{\phi} \Psi\right|^{2}+\partial_{\boldsymbol{x}} \Psi^{\dagger} \partial_{\boldsymbol{x}} \Psi+m_{\phi}^{2}|\Psi|^{2}+V_{\mathrm{eff}}(|\Psi|)\right] .
$$

The initial condition given in eq. (2.8) implies that this system is necessarily accompanied by a non-vanishing charge $Q$. Note that the factor $1 / 4$ should be included because we use the non-canonical kinetic term in eq. (2.15).

I-ball/oscillon as a projection of Q-ball. Let us seek the energetically favored configuration under a fixed charge $Q$, as in the case of Q-ball. Introducing a Lagrange multiplier $\omega$, all one has to do is to find the solution which minimizes $[18,55,56]$

$$
\begin{aligned}
I & =E+\omega\left(Q-\frac{1}{4} i \int_{\boldsymbol{x}}\left[\Psi^{\dagger}\left(\partial_{t} \Psi-i m_{\phi} \Psi\right)-\Psi\left(\partial_{t} \Psi^{\dagger}+i m_{\phi} \Psi^{\dagger}\right)\right]\right) \\
& =\frac{1}{4} \int_{\boldsymbol{x}}\left[\left|\partial_{t} \Psi-i\left(m_{\phi}-\omega\right) \Psi\right|^{2}+\partial_{\boldsymbol{x}} \Psi^{\dagger} \partial_{\boldsymbol{x}} \Psi+\left(m_{\phi}^{2}-\omega^{2}\right)|\Psi|^{2}+V_{\mathrm{eff}}(|\Psi|)\right]+\omega Q .
\end{aligned}
$$

\footnotetext{
${ }^{5}$ Then, the resultant effective potential is the same as one obtained from the so-called $\epsilon$-expansion. Thus, the $\epsilon$-expansion can be regarded as a perturbative computation of the effective potential in the nonrelativistic effective field theory, where derivatives acting on $\Psi$ are neglected. To make the comparison easier, we do not rescale $\Psi$ to make its kinetic term canonical.
} 
The minimization of the first term yields $\Psi(t, \boldsymbol{x})=e^{i \mu t} \psi(\boldsymbol{x}) / \sqrt{2}$ with $\mu \equiv m_{\phi}-\omega$. Without loss of generality, one can take $\psi(\boldsymbol{x})$ as real. Here note that the non-relativistic condition implies $\mu \ll m_{\phi}$. Since the pressure costs energy, let us assume a spherically symmetric solution [57]. Then, varying eq. (2.20) with respect to $\psi(r)$, one obtains the bounce equation:

$$
0=\left[\frac{\partial^{2}}{\partial r^{2}}+\frac{2}{r} \frac{\partial}{\partial r}\right] \psi(r)-\left(2 \mu m_{\phi}-\mu^{2}\right) \psi(r)-\frac{\partial V_{\mathrm{eff}}(\psi)}{\partial \psi} .
$$

From the finiteness of energy, the following boundary conditions are imposed:

$$
\lim _{r \rightarrow 0} \frac{\partial \psi(r)}{\partial r}=\lim _{r \rightarrow \infty} \psi(r)=0 .
$$

It can be regarded as an equation of motion for a one-dimensional system with a time variable $r$ and a friction $2 / r$. Therefore, a non-trivial solution may exist if the curvature of the "effective" potential at the origin, $-\left(2 \mu m_{\phi}-\mu^{2}\right)$, is negative and the "effective" potential, $-\left(\mu m_{\phi}-\mu^{2} / 2\right) \psi^{2}-V_{\text {eff }}$, becomes positive at $\psi \neq 0$. If this condition is fulfilled, the bounce solution may exist, where $\psi(\neq 0)$ starts to roll down its "effective" potential while the friction $2 / r$ dissipates its energy, and it stops at $\psi=0$ eventually. This condition can be expressed as

$$
0<\mu m_{\phi}-\frac{\mu^{2}}{2}<-\operatorname{Min}\left[\frac{V_{\mathrm{eff}}(\psi)}{\psi^{2}}\right]
$$

Once we find a family of solution for each $\mu$ as $\psi(r ; \mu)$, its charge and energy are obtained from

$$
\begin{aligned}
& Q(\mu)=\frac{1}{4} \omega \int 4 \pi r^{2} \mathrm{~d} r \psi^{2}(r ; \mu), \\
& E(\mu)=\frac{1}{4} \int 4 \pi r^{2} \mathrm{~d} r\left[\frac{1}{2} \omega^{2} \psi^{2}(r ; \mu)+\frac{1}{2}\left(\frac{\partial}{\partial r} \psi(r ; \mu)\right)^{2}+V_{\text {eff }}(\psi(r ; \mu))\right] .
\end{aligned}
$$

Here note again that $\omega=m_{\phi}-\mu$. The solution, $e^{-i \omega t} \psi(r ; \mu)$, is nothing but the Qball solution, which is a nontopological soliton in the presence of a conserved charge. In particular, it has been shown that [58]

$$
\frac{\partial E}{\partial Q}=\omega \equiv m_{\phi}-\mu
$$

Finally, let us relate this solution to the original scalar field $\phi$. Plugging this solution back to the definition given in eqs. (2.2) and (2.5), and omitting rapidly oscillating terms in $\delta \phi$, we obtain the I-ball/oscillon solution as a projection of the Q-ball solution:

$$
\phi(t, r ; \mu)=\Re\left[\frac{1}{\sqrt{2}} e^{-i\left(m_{\phi}-\mu\right) t} \psi(r ; \mu)\right]+\delta \phi_{V}[\psi(r ; \mu)],
$$

where the shift is determined perturbatively [see eq. (2.13)]

$$
\delta \phi_{V}[\psi(r ; \mu)]=-\frac{g_{3}}{2 m_{\phi}^{2}} \frac{\psi(r ; \mu)^{2}}{2}+\cdots .
$$

Therefore, the I-ball/oscillon can be regarded as a pseudo nontopological soliton associated with the approximate $\mathrm{U}(1)$ symmetry in the non-relativistic regime. In this sense, the Iball/oscillon can be seen as a projection of the Q-ball. 


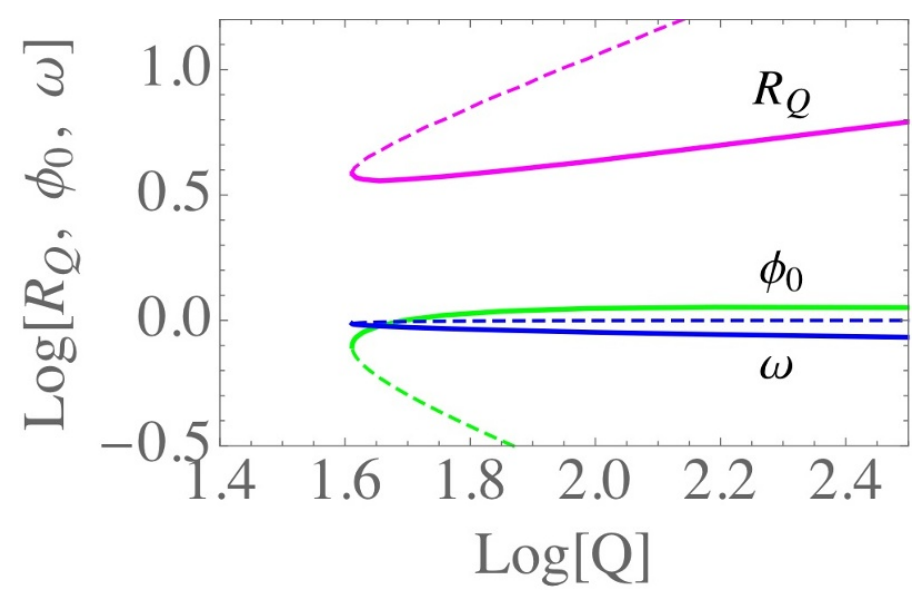

Figure 1. Radius $R_{Q}$ (magenta line), amplitude $\psi_{0}$ (green line), and parameter $\omega$ (blue line) of I-ball/oscillon as a function of charge $Q$. We assume $g_{4}=1$ and $g_{6}=0.4$. There are two solutions for a given charge when it is larger than a critical value $Q_{\mathrm{cr}} \simeq 10^{1.63}$. Solutions are stable against a small perturbation on one branch with thick lines, while they are unstable on the other branch with dashed lines.

Phase diagram and classical stability of Q-ball. Here we give a numerical example to clarify the properties of I-ball/oscillon explained so far. We assume $g_{3}=0$ in eq. (2.16) for simplicity. The configuration of $\psi(r ; \mu)$ can be calculated by solving eq. (2.21) for given values of $\mu$, where $V_{\text {eff }}$ is given by eq. (2.17). Then we can calculate the charge and energy of the configuration by eqs. (2.24) and (2.25), respectively. We also define a typical radius of the configuration by $\psi\left(r=R_{Q}\right)=\psi(r=0) / e$. All quantities are functions of $\mu$, so that we can rewrite the amplitude of the configuration $\psi(r=0)\left(\equiv \psi_{0}\right)$ and the radius $R_{Q}$ as a function of charge $Q$.

Figure 1 shows the phase diagram of the I-ball/oscillon in this theory, where we assume $g_{3}=1$ and $g_{6}=0.4$. We find that there is no solution of eq. (2.21) when its charge is less than a certain critical value $Q_{\mathrm{cr}} \simeq 1.63$. On the other hand, there are two solutions for a given charge $Q$ for $Q>Q_{\mathrm{cr}}$. On one branch, which is shown as thick lines in the figure, the amplitude of configuration and the radius increase as the charge increases, while on the other branch, which is shown as dashed lines in the figure, the amplitude decreases and the radius increases as the charge increases. The solutions on the former branch are stable against a small perturbation while the ones on the latter branch are unstable [52, 56, 59-61]. Therefore, we have only one stable solution for a given $Q\left(>Q_{\mathrm{cr}}\right)$. When the theory has a charge-violating interaction, as we discuss in the next subsection, the configuration evolves along with the stable branch as its charge decreases. Note that there is no solution for $Q<Q_{\mathrm{cr}}$, which implies that the I-ball/oscillon disappears when its charge decreases to the critical value $[37,40]$.

The unstable solutions may be understood physically in the following way. Suppose that the initial configuration is the corresponding Q-ball solution with a charge Q, whose energy is $E=\int^{Q} \mathrm{~d} Q^{\prime} \omega\left(Q^{\prime}\right)$ [see eq. (2.26)]. Recall here that the Q-ball is characterized by a total charge $Q$ and its frequency $\omega(Q)$. Then, let us perturb the solution with $\delta Q$. In order for the Q-ball solution to be stable against the perturbation, the energy of a Q-ball 
with charge $Q+\delta Q$ should be smaller than that of a Q-ball with charge $Q$ plus $\delta Q$ particles on it. Therefore, the following inequality should hold for the stability of Q-balls

$$
\int^{Q+\delta Q} \mathrm{~d} Q^{\prime} \omega\left(Q^{\prime}\right)<\int^{Q} \mathrm{~d} Q^{\prime} \omega\left(Q^{\prime}\right)+\delta Q \omega(Q) \quad \leftrightarrow \quad \frac{\partial \omega}{\partial Q}<0
$$

As can be seen from a phase diagram in figure 1, the branch with thick lines respects this inequality, while the other does not. In the next section, we will see that configurations close to the branch satisfying eq. (2.29) are actually long-lived. We also check numerically that those close to the other branch that violates eq. (2.29) decays immediately.

\subsection{Decay of I-ball/oscillon via U(1) breaking}

Now we are in a position to discuss the decay of I-ball/oscillon. In the previous section, we have shown that there is a spatially localized energetically favored solution by neglecting the U(1) breaking terms. First, let us discuss its typical property, and see that it necessarily contains a small amount of higher momentum modes.

To be specific, we characterize the bounce solution as follows:

$$
\psi(r) \simeq \psi_{0} e^{-r^{2} / 2 R^{2}}
$$

where $\psi_{0}$ is the amplitude at the center, and $R$ is a typical size of the bounce solution. The non-relativistic condition indicates that $m_{\phi} R \gg 1$. Thus, the momentum of the Iball/oscillon solution typically spreads over $k \lesssim 1 / R \ll m_{\phi}$, while its energy is concentrated on $k_{0}=\omega\left(=m_{\phi}-\mu\right)$ with $\mu \sim 1 / R \ll m_{\phi}$ as a delta function. The crucial observation is that it also contains a tiny amount of high momentum modes with $k \sim m_{\phi}$, nevertheless its energy is non-relativistic $k_{0}=m_{\phi}-\mu \simeq m_{\phi}$. The attractive effective potential enables these apparently "off-shell" modes to exist inside the I-ball/oscillon solution.

The existence of these modes alone does not spoil the stability of I-ball/oscillon, since they cannot propagate outside the I-ball/oscillon as free particles owing to the smallness of their energies [52]. However, in combination with the U(1) breaking terms, they play essential roles in production of relativistic modes. This is because we can hit the poles of relativistic modes such as eqs. (2.10) and (2.14) with the help of these high momentum modes. We refer this process as decay via spatial gradients, and discuss its nature by taking a simple example in the next subsection. Through this process, the I-ball/oscillon gradually reduces its energy/number, and eventually it exhibits sudden decay at a critically value of the charge, $Q_{\mathrm{cr}}$, below which there are no I-ball/oscillon solutions. See a numerical justification of this picture in section 3. It is noticeable that the energy spectrum of the I-ball/oscillon acquires a small width because the delta function in energy is broadened by the decay processes via U(1) breaking terms. Similar decay processes are discussed in the context of the axion star [62].

Decay via spatial gradients. As explained above, the I-ball/oscillon solution contains a tiny amount of high momentum modes though its energy is still non-relativistic. Performing the Fourier transform of eq. (2.30), one can explicitly see this property:

$$
\begin{aligned}
\psi(\boldsymbol{k}) & =\int_{\boldsymbol{x}} e^{-i \boldsymbol{k} \cdot \boldsymbol{x}} \psi(\boldsymbol{x}) \\
& \simeq \pi^{3 / 2} R^{3} \psi_{0} e^{-k^{2} R^{2} / 2},
\end{aligned}
$$


where we assume eq. (2.30) in the second line. There exist high momentum modes, $k \gtrsim m_{\phi}$, as a tail of the Gaussian distribution.

In the following, let us discuss how these high momentum modes induce the decay. To be concrete, we consider the following interaction term as an example:

$$
\mathcal{L}_{\text {int }}=\frac{g_{n+1}}{n+1} \phi^{n+1} .
$$

After splitting the scalar field into non-relativistic modes and the others, one finds the following term which contains a linear term of $\delta \phi$ :

$$
\begin{aligned}
\mathcal{L}_{\text {int }} & \supset \frac{g_{n+1}}{2^{3 n / 2}} \psi^{n} e^{-i n m_{\phi} t} \delta \phi+\text { H.c. } \\
& \equiv J(x) \delta \phi+\text { H.c. }
\end{aligned}
$$

which plays important roles in the classical decay.

Suppose that the I-ball/oscillon sits at the origin initially, and see how relativistic modes are produced in the presence of the I-ball/oscillon background. Let us emphasize that $\psi(r ; \mu)$ is now time-dependent because of the decay process. To estimate the decay rate in such a situation, first note that the imaginary part of effective action [see eq. (2.15)] contributes to the time-dependence of the amplitude $\psi(r, t ; \mu)$ :

$$
\begin{aligned}
& \frac{1}{4}\left[\left(2 m_{\phi} i \frac{\partial}{\partial t}+\frac{\partial^{2}}{\partial t^{2}}+\nabla^{2}\right) \Psi(t, x)-\frac{\partial V_{\text {eff }}}{\partial \Psi^{\dagger}}\right]-i \frac{\partial \Gamma}{\partial \Psi^{\dagger}}=0 \\
& \leftrightarrow \frac{1}{2} m_{\phi} i \frac{\partial}{\partial t} \psi(r, t ; \mu)-i \frac{\partial \Gamma}{\partial \psi}=0,
\end{aligned}
$$

where we use the equation of motion of the real part in the second line and neglect a term proportional to $\partial^{2} \psi / \partial t^{2}$ because the decay process is assumed to be much slower than the oscillation time scale $m_{\phi} \cdot{ }^{6}$ Recalling the definition of the charge given in eq. (2.24), one finds its time derivative as follows:

$$
\dot{Q}=\frac{1}{2} \omega \int_{\boldsymbol{x}} \psi \dot{\psi} \simeq+\int 4 \pi r^{2} \mathrm{~d} r \psi \frac{\partial \Gamma}{\partial \psi} .
$$

The imaginary part of effective action $i \Gamma$ comes from diagrams like eqs. (2.10) and (2.14). For the interaction of eq. (2.35), it is given by

$$
-i \frac{\partial \Gamma}{\partial \Psi^{\dagger}} \supset i \Im\left[\frac{\partial J^{\dagger}(x)}{\partial \Psi^{\dagger}} \int_{y} G_{\mathrm{ret}}(x, y) J(y)\right] .
$$

Here $G_{\text {ret }}(x, y)$ is the retarded propagator for relativistic modes $\delta \phi$, which satisfies: ${ }^{7}$

$$
\left(\square_{x}+m_{\phi}^{2}\right) G_{\text {ret }}(x, y)=\delta(x-y) .
$$

\footnotetext{
${ }^{6}$ And in fact, we will see soon that the decay rate is much smaller than $m_{\phi}$ a posteriori.

${ }^{7}$ Here we have neglected a small shift of the mass, $m_{\phi}$, in the presence of the I-ball/oscillon background. The effective mass changes according to the spatial gradient of the I-ball/oscillon. The non-relativistic condition indicates that the gradient is much larger than $1 / m_{\phi}$. Since we are interested in relativistic modes which has energy and momentum comparable to or larger than $m_{\phi}$, we may use this approximation.
} 
It is convenient to consider it in the momentum space: ${ }^{8}$

$$
\int_{y} G_{\mathrm{ret}}(x, y) J(y)=\int_{K} G_{\mathrm{ret}}(K) J(K) e^{-i K \cdot x},
$$

where

$$
\begin{aligned}
\Im G_{\text {ret }}(k) & =\frac{\pi}{2 \omega_{k}}\left[\delta\left(k_{0}-\omega_{k}\right)-\delta\left(k_{0}+\omega_{k}\right)\right] \\
J(K) & =(2 \pi) \delta\left(k_{0}-n \omega\right) \tilde{J}_{n+1}(\boldsymbol{k}) \\
\tilde{J}_{n+1}(\boldsymbol{k}) & \equiv \frac{g_{n+1}}{2^{3 n / 2}} \int_{\boldsymbol{x}} e^{-i \boldsymbol{k} \cdot \boldsymbol{x}} \psi^{n}(\boldsymbol{x}) .
\end{aligned}
$$

Thus we have the imaginary part of the following term in the equation of motion for $\Psi$ :

$$
\frac{\partial \Gamma}{\partial \psi(x)} \supset-\Im\left[\frac{2 n g_{n+1}}{2^{3 n / 2}} \psi^{n-1}(x) e^{i n m_{\phi} x_{0}} \int_{K} G_{\mathrm{ret}}(K) J(K) e^{-i K \cdot x}\right] .
$$

The imaginary part corresponds to the production of on-shell relativistic modes outside the I-ball/oscillon. Plugging the I-ball/oscillon solution into $\psi$, one can estimate its decay rate perturbatively. After some algebras, we finally obtain the decay rate of the charge in the case of eq. (2.35):

$$
\begin{aligned}
\dot{Q} & =-2 \pi n \int_{\boldsymbol{k}}\left|\tilde{J}_{n+1}(\boldsymbol{k})\right|^{2} \delta\left(n^{2} \omega^{2}-m_{\phi}^{2}-|\boldsymbol{k}|^{2}\right) \\
& \simeq-\frac{n}{2 \pi} \sqrt{n^{2}-1} \omega\left|\tilde{J}_{n+1}\right|^{2}
\end{aligned}
$$

where $\tilde{J}_{n+1}$ is given by eq. (2.44) with $|\boldsymbol{k}|=\sqrt{n^{2} \omega^{2}-m_{\phi}^{2}} \simeq \sqrt{n^{2}-1} \omega$. In the second line, we use $\psi(\boldsymbol{x})=\psi(r)$ and $\tilde{J}_{n+1}(\boldsymbol{k})=\tilde{J}_{n+1}(|\boldsymbol{k}|)$. This is the emission rate of particles with a momentum of $|\boldsymbol{k}|=\sqrt{n^{2} \omega^{2}-m_{\phi}^{2}} \simeq \sqrt{n^{2}-1} \omega$ via the $n+1$-point interaction.

Once we assume the configuration as the Gaussian form of eq. (2.30), we can analytically calculate $\tilde{J}(\boldsymbol{k})$ and obtain

$$
\dot{Q}=-C(n) \tilde{g}_{n+1}^{2} \epsilon^{3(n-2)} \exp \left(-\frac{n^{2}-1}{n} \frac{1}{\epsilon^{2}}\right) \omega \times Q^{n}
$$

where the order one factor $C(n)$ are defined as

$$
C(n) \equiv \frac{\sqrt{n^{2}-1}}{2^{n-2} \pi^{3 n / 2-2} n^{2}} .
$$

Here we have defined a dimensionless coupling, $\tilde{g}_{n+1} \equiv g_{n+1} \omega^{n-3}$, and the epsilon parameter, $\epsilon \equiv 1 / \omega R$. As can be inferred from eq. (2.45), this process corresponds to the production of relativistic modes with $k_{0} \simeq n \omega$ and $k \simeq \sqrt{n^{2}-1} \omega$ owing to the combination of high momentum modes inside the I-ball/oscillon and the $\mathrm{U}(1)$ breaking interaction terms. Note that the non-relativistic condition indicates that the epsilon parameter should

\footnotetext{
${ }^{8}$ Here and hereafter, we have used the shorthanded notation: $\int_{k} \equiv \int \mathrm{d}^{4} k /(2 \pi)^{4}$ and $\int_{\boldsymbol{k}} \equiv \int \mathrm{d}^{3} k /(2 \pi)^{3}$.
} 
be smaller than unity, $\epsilon \ll 1$, which ensures that the decay rate is exponentially suppressed. Also, note that the rate becomes exponentially smaller for a larger $n$, which is confirmed in our full numerical simulation as we explain in the next section. (See also refs. [26, 36$38,52]$ for instance.) This implies that the cubic term $(n=3)$ makes the life time of the I-ball/oscillon shorter than that of the $\mathbb{Z}_{2}$ invariant case.

Toy model and its implications. Here we apply the above theory of decay process to a toy model. Let us consider a Q-ball in a $U(1)$ theory with the potential of eq. (2.17). Once we specify the coupling constants, all properties of Q-ball are determined as a function of its charge $Q$. Then we impriment its time evolution by imposing eq. (2.47) by hand. This is a toy model that simply describes the time evolution of I-ball/oscillon via the decay process discussed above. One can see that the qualitative result of this toy model is consistent with the result of full numerical simulation of I-ball/oscillon that we explain in the next section.

For a given charge $Q$, we can calculate its configuration $\psi(r)$ and then we can calculate $\tilde{J}_{n}$ numerically. Figure 2 shows the value of $\tilde{J}_{n} / g_{n}$ as a function of charge $Q$ for $n=3$, 4 , and 6. We are interested in solutions on the stable branch, which is shown as solid blue lines in the figure. We confirm that typical values of $\tilde{J}_{n} / g_{n}$ is exponentially smaller for a larger $n$, which is explicitly shown above in the Gaussian profile approximation as eq. (2.48). We can see that $\tilde{J}_{n} / g_{n}$ oscillates as a function of charge and it vanishes at certain values. This implies that the decay process becomes inefficient for a solution with charges around these values. For example, suppose that the initial charge of I-ball/oscillon is $10^{2.4}$. If the three point interaction is absent, that is, if $g_{3}=0$, its charge decreases mainly via the four point interaction. However, when its charge decreases to $10^{2.25}, \tilde{J}_{4}^{2}$ decreases to 0 and its decay rate is suppressed accordingly. This implies that I-ball/oscillon reaches an attractor solution in this toy model. In realistic, we also have a six-point interaction because of $g_{6}=0.4$, which gives nonzero decay rate at the critical value though it is exponentially suppressed. Therefore, the attractor solution is not exactly stable and the charge can decrease below the point of $\tilde{J}_{4}=0$. This can be seen in the lower right panel in the figure, where we plot the value of $-d Q / d t$ with the contributions of four and six point interactions. Although the typical value of decay rate is of order $10^{-2}$, it is as small as $10^{-8}$ but is still nonzero at the attracter points. Eventually, the solution goes away from the attractor point and then $\tilde{J}_{4}$ increases and the decay rate increases as shown in the figure. We can see that there are also points at which $\tilde{J}_{4}=0$ around $Q=10^{1.94}$ and $Q=10^{1.64}$. This implies that the time-evolution of charge has many bumps and attractor region due to these properties. Note that there is a point at which $\tilde{J}_{4}=0$ just above the critical point $Q_{\text {cr. }}$. This implies that the I-ball/oscillon reaches an attractor and then suddenly decays due to the classical instability. This feature is checked numerically as we explain in the next section.

At each time step, we can calculate its configuration for a given charge $Q$. Then we can calculate $\tilde{J}_{n}$ numerically and thus we obtain its decay rate eq. (2.47), which determines charge in the next time step. In this way, we can obtain the time evolution of these quantities. Figure 3 shows the time evolution of charge $Q$ in this toy model, where we assume $g_{3}=0$ and the initial value of its charge is taken to be $10^{2.2}$. Since the initial 

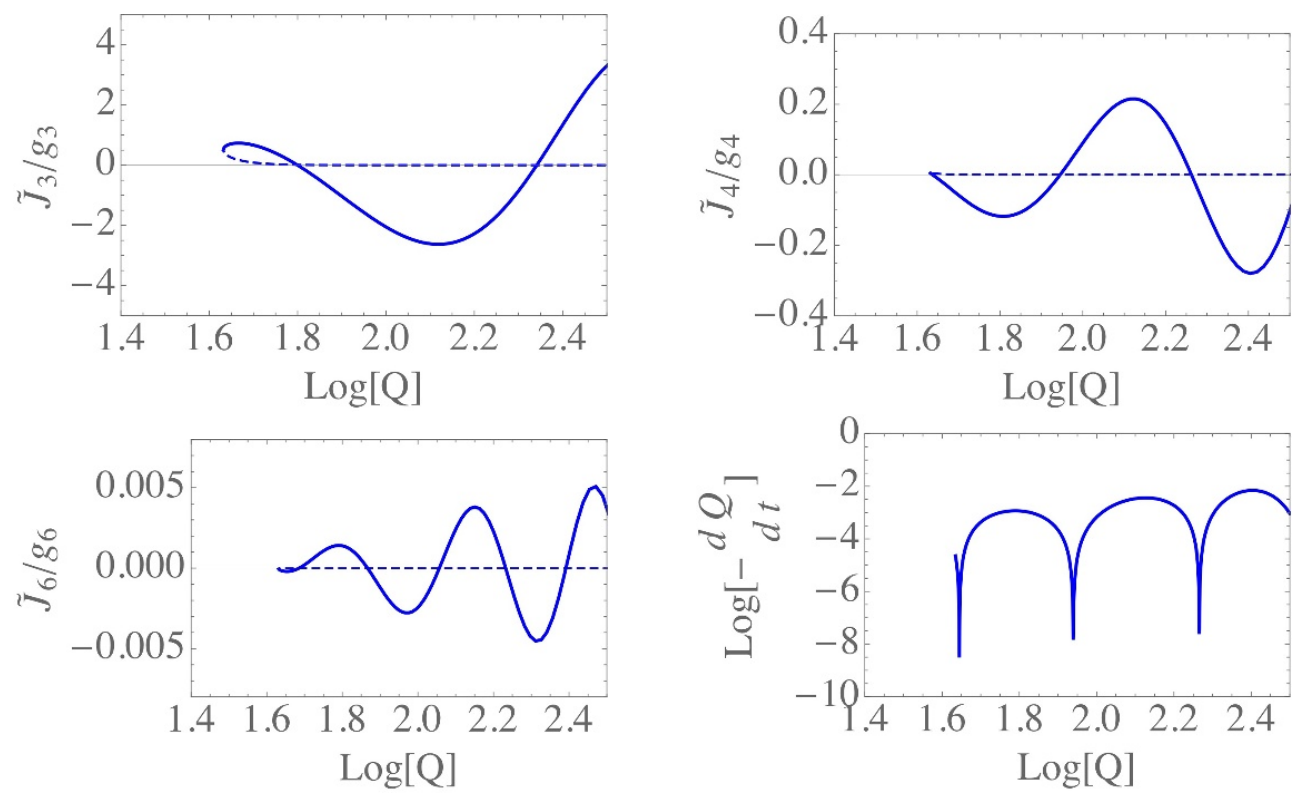

Figure 2. Values of $\tilde{J}_{n} / g_{n}$ as a function of charge $Q$ for $n=3$ (upper left panel), $n=4$ (upper right panel), and $n=6$ (lower left panel), and the sum of $-d Q / d t$ (lower right panel). We assume $g_{4}=1$ and $g_{6}=0.4$.

charge is smaller than $10^{2.25}$, it decreases fast due to the four-point interaction. Then it reaches to an attractor point, at which the charge is about $10^{1.94}$. After that, the decay rate is suppressed as low as $10^{-8}$ but is still nonzero (see figure 2). Then it escapes from the attractor point and its charge decreases again due to the four-point interaction. Then it reaches the second attractor point at $Q \simeq 10^{1.64}$, which is just above the critical value $Q_{\mathrm{cr}} \simeq 10^{1.63}$. This means that there is some time before the I-ball/oscillon disappears due to the classical instability. We compare these results with the full numerical simulation of the evolusion of I-ball/oscillon in the next section.

\section{Numerical simulation}

We perform numerical simulations to solve the equation of motion of a real scalar field in $3+1$ dimensions, assuming spherical symmetry to reduce the spacial dependence in 3 dimensions to a radial dependence. In this section, we show our results of numerical simulations that justify our theory discussed in the previous section.

We can rescale parameters and variables such as

$$
\begin{aligned}
t & \rightarrow t / m_{\phi} \\
\phi & \rightarrow \phi / \sqrt{g_{4}} \\
r & \rightarrow r / m_{\phi}
\end{aligned}
$$

to eliminate $m_{\phi}$ and $g_{4}$ in the real scalar field theory. In other words, we can use units of $m_{\phi}=1$ and $g_{4}=1$ in eq. (2.16) without loss of generality. The equation of motion for a 


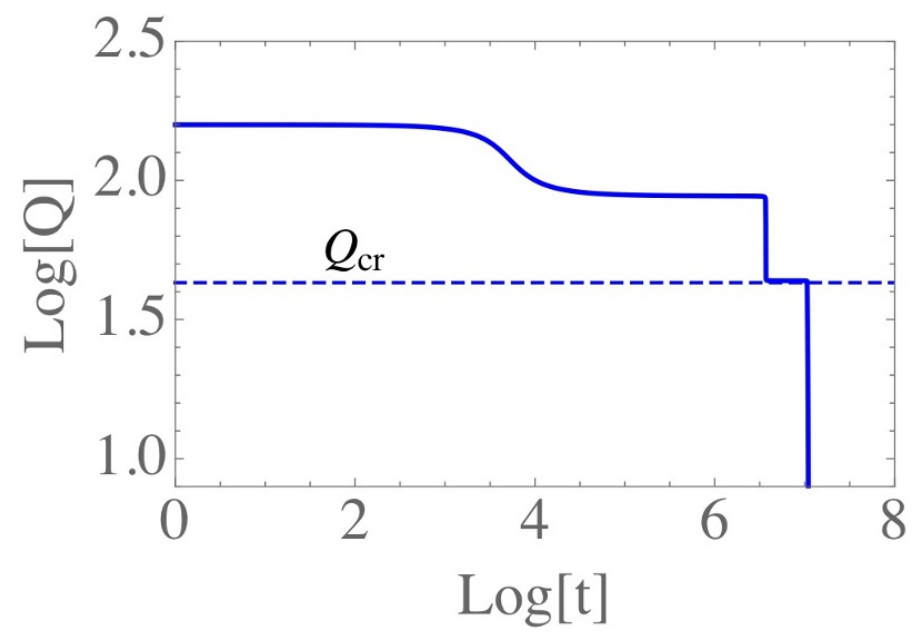

Figure 3. Time evolution of charge $Q$ in the toy model. We assume $g_{4}=1$ and $g_{6}=0.4$. The blue dashed line represents the critical value $Q_{\mathrm{cr}} \simeq 10^{1.63}$ below which there is no solution of I-ball/oscillon.

scalar field with a polinomial potential is then given by

$$
\begin{aligned}
\frac{\mathrm{d}^{2}}{\mathrm{~d} t^{2}} \phi-\frac{\mathrm{d}^{2}}{\mathrm{~d} r^{2}} \phi-\frac{2}{r} \frac{\mathrm{d}}{\mathrm{d} r} \phi+\frac{\partial}{\partial \phi} V_{\text {num }}(\phi) & =0, \\
V_{\text {num }}(\phi) & =\frac{1}{2} \phi^{2}+\frac{1}{3} g_{3} \phi^{3}-\frac{1}{4} \phi^{4}+\frac{1}{6} g_{6} \phi^{6},
\end{aligned}
$$

where we assume spherical symmetry and consider the dynamics of radial direction. Here we redefine $g_{3}$ and $g_{6}$ to absorb $m_{\phi}$ and $g_{4}$ after rescaling the variables and the field value.

We develop a numerical lattice code to solve the classical equation of motion and investigate the time-evolution of I-ball/oscillon. We use the 6th order leapfrog method for time-evolution. We take the lattice grid number as $N=10^{3}$ and the lattice volume as $L=50$ (in the unit of $m_{\phi}=1$ ), which is much larger than a typical size of I-balls/oscillons. The grid size is thus given by $\Delta x=L / N=0.05$. We take each time step as $\Delta t=0.01$ and numerical simulation is performed from $t=0$ to $t=T_{\max }=10^{5}$. We check that our results of numerical simulations are rarely dependent on these numerical setups. For example, the heights and peak locations in figure 5 do not change when we change $N, L$, and/or $\Delta t$ by amounts of $50 \%$.

The boundary condition at $r=0$ is taken to be $\partial \phi / \partial r(r=0)=0$ while the one at the other boundary (i.e., at $r=L$ ) is taken to be the absorbing boundary condition (see appendix A in ref. [42])..$^{9}$ The absorbing boundary condition allows us to investigate the time-evolution of I-ball/oscillon less affected by the boundary effect at $r=L$ because emitted particles from the I-ball/oscillon are absorved and are rarely reflected by that boundary. We are interested in the time-evolution of I-balls/oscillons after they form.

\footnotetext{
${ }^{9}$ There is a typo in eq. (A9) in ref. [42]. The sign of the third tern $-1 / 2 m^{2} \varphi$ should be positive for a correct absorbing boundary condition.
} 
Thus, initial conditions are taken to be a spacially-localized gaussian configuration:

$$
\phi(t=0, r)=\phi_{\mathrm{ini}} e^{-r^{2} / R_{\mathrm{ini}}^{2}},
$$

and $\dot{\phi}=0$, where $\phi_{\text {ini }}$ and $R_{\text {ini }}$ are some constants specified below.

Note that some important quantities discussed in the previous section, such as $Q$ and $R_{Q}$, cannot be directly deduced from our numerical simulation because they are defined by $\Psi$ (not $\phi$ ) [see eq. (2.24)]. In order to compare our numerical results with our theory discussed in the previous seciton, we approximate them by taking time-avarage over some period of oscillation-time scale $m_{\phi}^{-1}$. For example, $Q$ is approximated by $\bar{Q}$ :

$$
\begin{aligned}
\bar{Q} & =\frac{1}{T_{\text {avl }}} \int_{t-T_{\text {avl }}}^{t} \mathrm{~d} t \int \mathrm{d}^{3} x \frac{1}{2} \dot{\phi}^{2} \\
& =\frac{1}{T_{\text {avl }}} \int_{t-T_{\text {avl }}}^{t} \mathrm{~d} t \int 4 \pi r^{2} \frac{1}{2} \dot{\phi}^{2} \mathrm{~d} r,
\end{aligned}
$$

and $R_{Q}$ is approximated by $\bar{R}_{Q}$ :

$$
\begin{aligned}
\bar{\phi}\left(r=\bar{R}_{Q}\right) & =\bar{\phi}(r=0) / e, \\
\bar{\phi}(t, r) & \equiv\left[\frac{1}{T_{\text {avl }}} \int_{t-T_{\text {avl }}}^{t} \mathrm{~d} t \phi^{2}(t, r)\right]^{1 / 2},
\end{aligned}
$$

where $T_{\text {avl }}$ is a duration time for time-avarage. We replace the integral by a summation in our numerical simulations. We take $T_{\text {avl }}=10$ in our numerical simulation. On the other hand, the energy is defined by $\phi$ such as eq. (2.19) without taking time-avarage.

\subsection{Case with $\mathbb{Z}_{2}$ symmetry}

Here we show our results of numerical simulation for the case with $\mathbb{Z}_{2}$ symmetry (i.e., for the case of $\left.g_{3}=0\right)$. We take $g_{6}=0.4$ as an example. Figures 4 show the time-evolution of $\phi_{0}, \bar{R}_{Q}, E$, and $\bar{Q}$ as a function of time for $R_{\text {ini }}=10$ (upper left panel), $R_{\text {ini }}=7$ (upper right panel), and $R_{\text {ini }}=5$ (lower left panel), and the contours of $\phi_{0}$ and $\bar{R}_{Q}$ as a function of $\bar{Q}$ (lower right panel). Here, $\phi_{0}$ is the time-averaged field value at $r=0$ (i.e., $\left.\phi_{0} \equiv \bar{\phi}(r=0)\right)$ and $\bar{R}_{Q}$ is a typical size of I-ball/oscillon defined by eq. (3.9). We take $\phi_{\text {ini }}=1$. We divide the time interval $\left[0, T_{\max }\right]$ by $10^{4}$ segments and take average over each segment to calculate $\phi_{0}, \bar{R}_{Q}$, and $\bar{Q}$ (i.e., we take $T_{\text {avl }}=T_{\max } / 10^{4}=10$.) This is the reason that the plot starts at $t=T_{\max } / 10^{4}=10$ in the figures.

From the upper left panel, we can see that the averaged charge $\bar{Q}$ reaches an attractor point and the I-ball/oscillon is too long-lived to calculate its time evolution numerically until it reaches the critical point. Although the I-ball/oscillon in the upper right panel starts from an averaged charge below that attractor point, it reaches another attractor point and again it is long-lived. The I-ball/oscillon in the lower left panel starts from an averaged charge below the latter attractor point and can reaches the critical value, at which the I-ball/oscillon suddenly decay due to the classical instability.

We take some initial conditions by changing $R_{\text {ini }}$ to find time-evolutions of these parameters in $\phi_{0}-\bar{Q}$ and $\bar{R}_{Q}-\bar{Q}$ planes (the lower right panel in figure 4 ). We also plot 

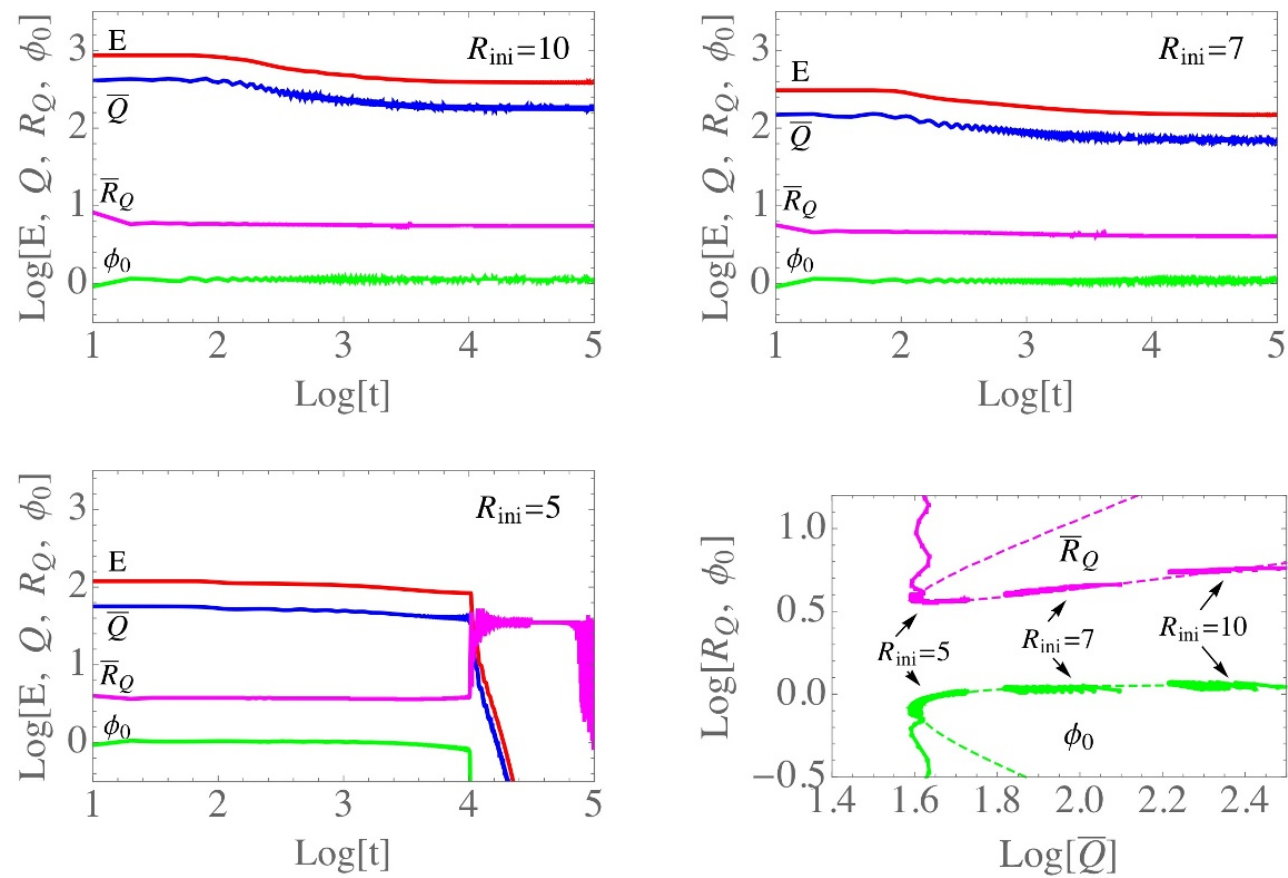

Figure 4. Time-evolution of $E$ (red line), $\bar{Q}$ (blue line), $\phi_{0}$ (green line), and $\bar{R}_{Q}$ (magenta line) as a function of time for $R_{\text {ini }}=10$ (upper left panel), $R_{\text {ini }}=7$ (upper right panel), $R_{\text {ini }}=5$ (lower left panel), and that of $\phi_{0}$ (green line) and $\bar{R}_{Q}$ (magenta line) as a function of $\bar{Q}$ for $R_{\text {ini }}=5,7,10$ (lower right panel). In the lower right panel, we also plot theoretical lines of $\phi_{0}$ (green dashed line) and $R_{Q}$ (magenta dashed line). We take $g_{6}=0.4$ and $\phi_{\text {ini }}=1$. We also plot theoretical lines of $R_{Q}$ and $\phi_{0}$ (i.e., $\left.\psi_{0}\right)$ as a function of $Q$ (dashed lines), which are computed as explained in the previous section [See also eq. (2.17) and eq. (2.24)].

theoretical lines of $R_{Q}$ and $\phi_{0}$ (i.e., $\psi_{0}$ ) as a function of $Q$, which are calculated by the procedure discussed in the previous section with eq. (2.17) [see also eq. (2.24)]. One can see that our predictions are consistent with the results of numerical simulations. Even if we take different value of $R_{\text {ini }}$, values of $\bar{R}_{Q}$ and $\phi_{0}$ soon reach the theoretical lines (before $\left.t=T_{\max } / 10^{3}\right)$ and then evolve along with these lines. In particular, once $\bar{Q}$ decreases down to a critical point, which is defined by $\partial \omega / \partial Q=0$ [see eq. (2.29)], the I-ball/oscillon suddenly decays.

We also plot spectra of particles in figure 5, where the red line represents the spectrum of energy flux from the I-ball/oscillon and the blue one represents that inside the I-ball/oscillon. The spectrum is deduced from the numerical simulations by extracting field configurations in a certain time interval $\left[t_{1}-N_{1} \Delta t, t_{1}\right]$ at a certain radius $r_{1}(=\Delta x$ or $L$ ) and taking Fourier transformation such as

$$
j_{\text {flux }}\left(k_{0}\right)=4 \pi r_{1}^{2} v \frac{1}{2} k_{0}^{2} \tilde{\phi}^{2}\left(k_{0}\right)
$$

where $k_{0}=\left(k^{2}+1^{2}\right)^{1 / 2}$ is energy, $k$ is momentum, $v=k / k_{0}$ is velocity, and $\tilde{\phi}\left(k_{0}\right)$ is 


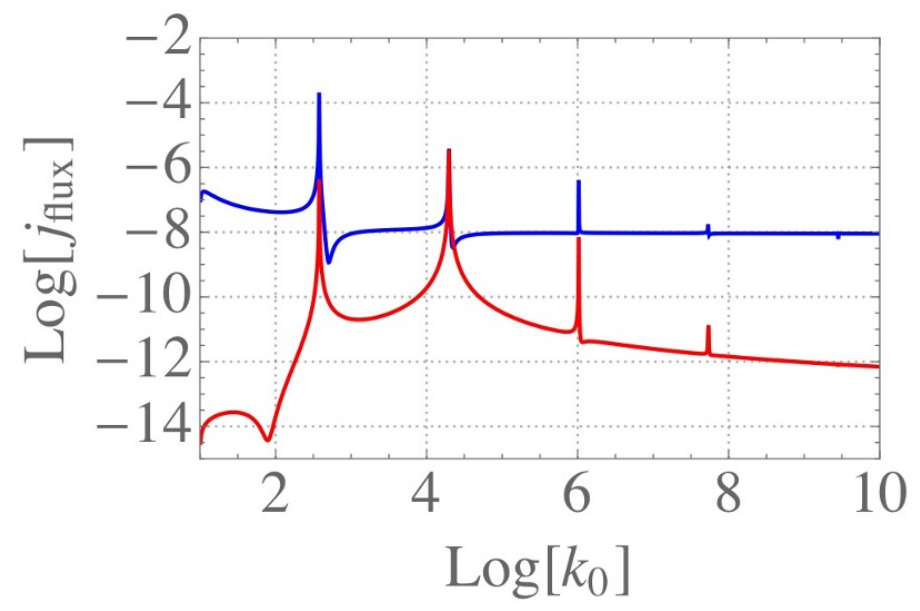

Figure 5. Spectra of energy flux outside I-ball/oscillon (red line) and inside I-ball/oscillon (blue line) at the time of $t_{1}=10^{5}$ for the case of $g_{6}=0.4$ and $R_{\text {ini }}=10$.

Fourier-tramsformed configuration calculated by

$$
\tilde{\phi}\left(k_{0}\right)=\frac{1}{N_{1}} \sum_{i=1}^{N_{1}} \phi\left(t=t_{1}-N_{1} \Delta t+(i-1) \Delta t, r=r_{1}\right) e^{2 \pi i(i-1)(j-1) / N_{1}}
$$

with $k_{0}=2 \pi(j-1) /\left(N_{1} \Delta t\right)\left(j=1,2,3, \ldots, N_{1}\right)$. In the figure, we take $t_{1}=T_{\max }=10^{5}$ and $N_{1} \Delta t=10^{3}$. Note that the unit energy that each "particle" inside I-ball/oscillon has is not $m_{\phi}$ but $\omega\left(=m_{\phi}-\mu\right)$ [see eq. (2.26)], which is of order but less than $m_{\phi}$. One can see that I-balls/oscillons dominantly emit relativistic particles with the energy of $(2 n+1) \omega$ $(n=1,2,3, \ldots)$ in the case with $\mathbb{Z}_{2}$ symmetry. This is just the process we discuss in section 2.3. Note that we plot the spectrum of particle at the time of $t=T_{\max }$, at which the I-ball/oscillon stays at the attractor point. This implies that the four-point interaction process is suppressed and the dominant contribution is due to the six-point interaction. This is explicitly shown in the figure, where the dominant contribution of flux is the particle with the energy of $5 \omega$.

\subsection{Case without $\mathbb{Z}_{2}$ symmetry}

Next we show our results of numerical simulation for the case without $\mathbb{Z}_{2}$ symmetry. Figures 6 show the time-evolution of $\phi_{0}, \bar{R}_{Q}, E$ and $\bar{Q}$ as a function of time (left panel) and $\phi_{0}$ and $\bar{R}_{Q}$ as a function of $\bar{Q}$ (right panel). We take $\phi_{\text {ini }}=1, R_{\text {ini }}=7, g_{6}=0.4$, and $g_{3}=0.01,0.02,0.05,0.1$ as examples. One can see that the $\mathbb{Z}_{2}$ breaking term affects the evolution of I-ball/oscillon and makes I-ball/oscillon decay fast. Note that the averaged charge of I-ball/oscillon has approximated attractor points for the case of $g_{3}=0.01$ (and 0.02 ), while there are no such points for $g_{3}=0.05$ and 0.1 . This implies that the dominant decay process is the four-point interaction for the former case while it is the three-point interaction for the latter case.

Figure 7 shows plots of energy flux from I-balls/oscillons at the time of $t_{1}=5 \times 10^{3}$. The $\mathbb{Z}_{2}$ breaking term leads to decay modes with even units of energy, such as $2 \omega$. For 

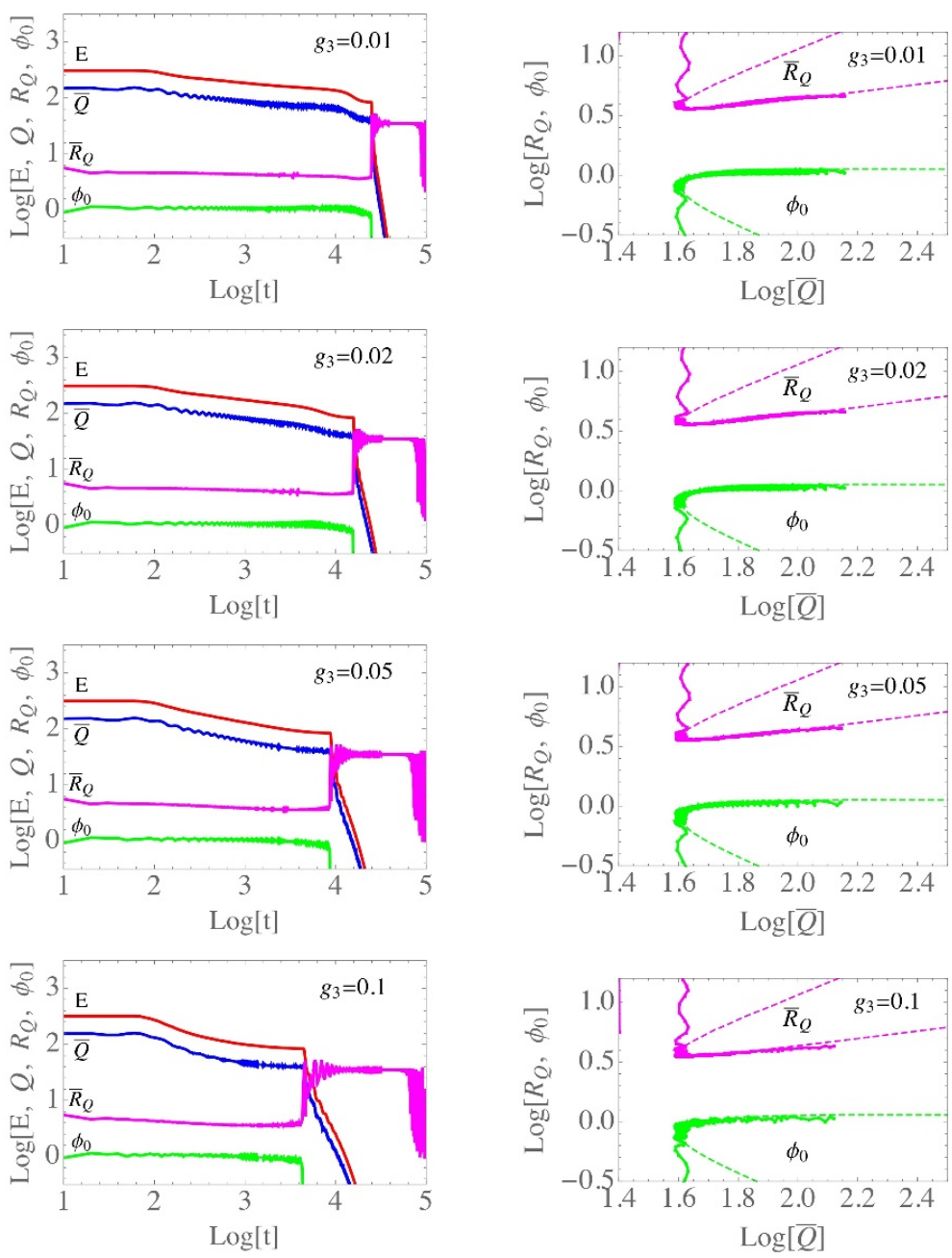

Figure 6. Same as figure 4 but with $g_{3}=0.01,0.02,0.05,0.1$.

$g_{3} \lesssim 0.01$, the energy flux of even modes is less than that of $3 \omega$, while for larger $g_{3}$, the energy flux of even modes is comparable or larger than that of $3 \omega$. One can also see that the height of $2 \omega$ mode is roughly proportional to $g_{3}^{2}$ for $g_{3} \lesssim 0.01$, which justifies eq. (2.47) [see also eq. (2.48)]. At the time of $t_{1}=5 \times 10^{3}$, the averaged charge of the I-ball/oscillon is just above the critical value for the case of $g_{3}=0.1$. This may be the reason why the flux is dominated by the non-relativistic mode for $g_{3}=0.1$.

\subsection{Case of double well potential}

Finally, we consider a real scalar field theory with a double well potential, which is widely studied in the literature. It has a $\mathbb{Z}_{2}$ symmetry that is spontaneously broken at the minimum of the potential. Thus, this case falls into "without $\mathbb{Z}_{2}$ symmetry" in our classification. The potential is given by

$$
V(\phi)=\frac{1}{4}\left[\left(\phi-\frac{\sqrt{2}}{2}\right)^{2}-\frac{1}{2}\right]^{2},
$$



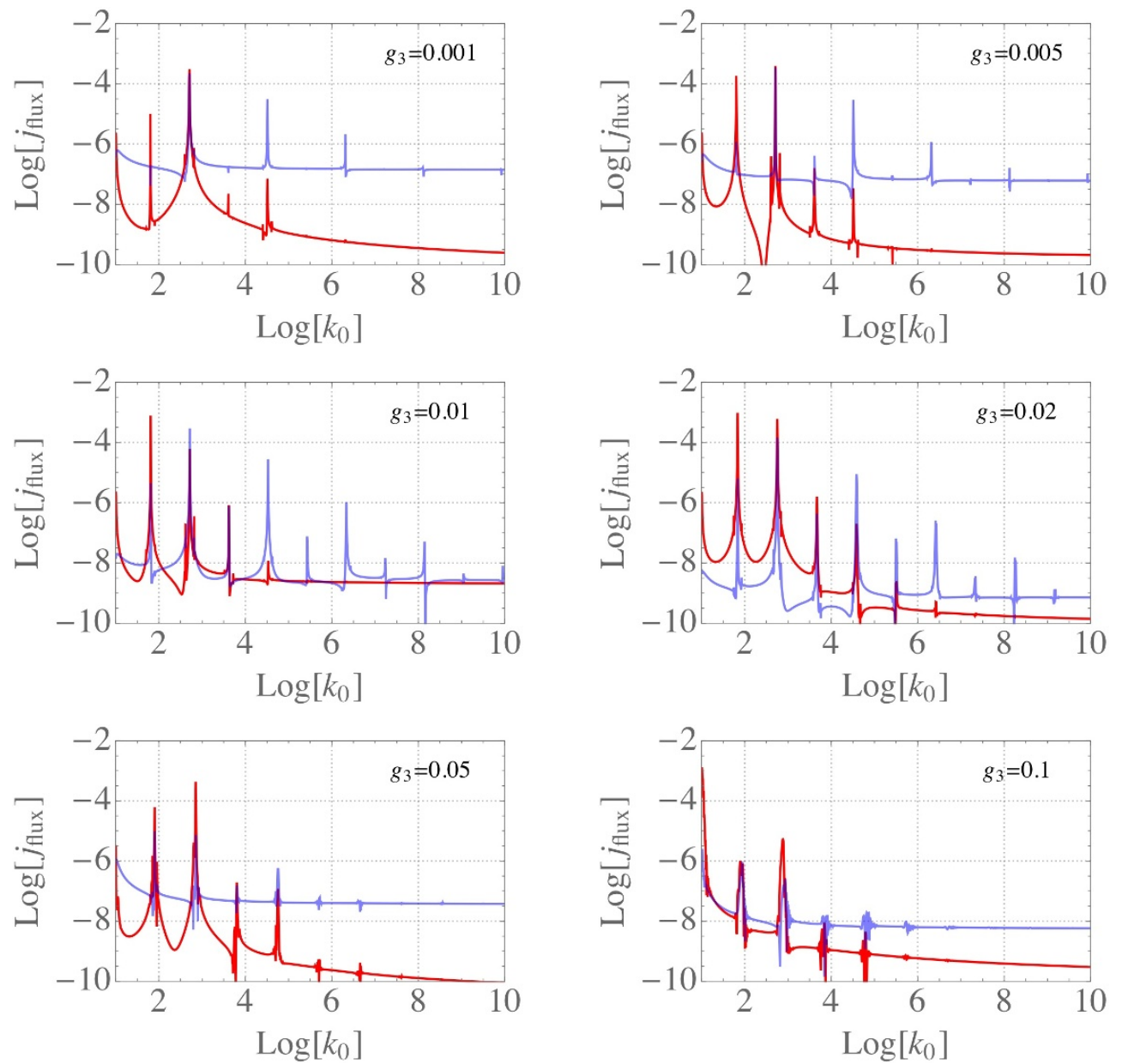

Figure 7. Same as figure 5 but with $g_{3}=0.001,0.005,0.01,0.02,0.05,0.1$.

which is a particular case of the one without $\mathbb{Z}_{2}$ symmetry such as $g_{3}=3 \sqrt{2} / 2, g_{4}=1$, and $g_{6}=0$ in eq. (2.16). Note that we take $\phi=0$ at the minimum of the potential. In this case, the I-ball/oscillon is relatively short lived, so that we take $T_{\max }=10^{4}$ (with $T_{\text {avl }}$ fixed) in our numerical simulation. We integrate eq. (3.7) from $r=0$ to 10 so that emitted particles are not included.

We take $\phi_{\text {ini }}=-1$ and $R_{\text {ini }}=7$ for the initial condition. The resulting time-evolution of $\phi_{0}, \bar{R}_{Q}, E$, and $\bar{Q}$ are plotted in the left panel in figure 8 . The values of $\phi_{0}$ and $\bar{R}_{Q}$ are plotted as a function of $\bar{Q}$ in the right panel in figure 8. We also plot theoretical results as dashed lines, where we include up to eighth order terms in the effective potential and the leading order correction of the difference between $m_{\phi}$ and $\omega\left(=m_{\phi}-\mu\right)$ (see appendix B). We take into account a leading order derivative correction up to mass dimension ten. The consistency with the theoretical result is not so good as the previous examples because higher-order terms in the effective potential may be relevant when $g_{3}=\mathcal{O}(1)$. However, qualitative features are still consistent with the numerical simulation. In particular, Iball/oscillon suddenly decay when $\bar{Q}$ decreases down to a certain critical value. 

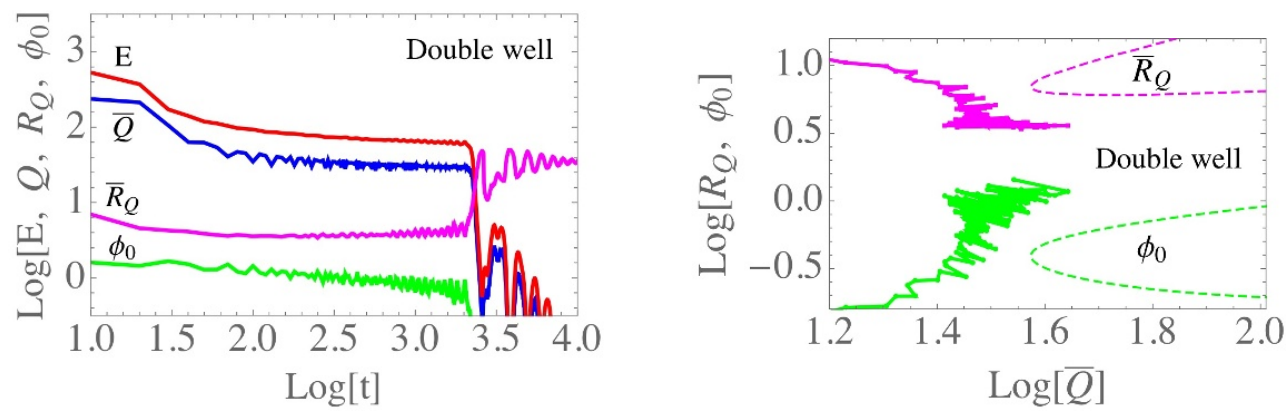

Figure 8. Same as figure 4 but for a theory with the double well potential. We take $\phi_{\text {ini }}=-1$ and $R_{\text {ini }}=7$.

\section{Conclusions and discussion}

In this paper, we have investigated the reason for the longevity of the I-balls/oscillons in real scalar field theories, which is apparently mysterious because there are no evident conserved charges. The point is that these localized configurations can be well described non-relativistically. As a result, an approximate symmetry emerges in that regime, which reflects the approximate number conservation, although the number is not conserved relativistically. The longevity of I-balls/oscillons can be understood as the smallness of the breaking of this approximated symmetry.

To clarify this viewpoint, we have explicitly constructed an effective theory for nonrelativistic dynamics by integrating out relativistic modes. The resulting effective theory comes to have an approximate global U(1) symmetry, whose charge is the number of particles. The breaking of $\mathrm{U}(1)$ symmetry is encoded in the imaginary part of this effective action, which indicates the production of relativistic particles via number violating processes. We have shown that the profile of I-balls/oscillons can be obtained as far as the effect of the imaginary part can be safely neglected. Therefore, the profile of the I-balls/oscillons can be viewed as a projection of Q-balls onto the one axis (e.g., real part of a complex scalar field $\left.\Phi_{Q}\right)$ in the field space. We have demonstrated how to obtain a family of Iballs/oscillons as a function of the charge $Q$ for a given potential, and clearly shown that there exists a critical value of the charge, $Q_{\mathrm{cr}}$, below which we do not have stable solutions.

Then, we discuss the classical decay of I-balls/oscillons. As in the case of computing a decay rate of unstable particles, we can estimate the decay rate of I-balls/oscillons, by first regarding them as stable and then computing the imaginary part perturbatively. Since we are interested in the classical decay, we omit all the loop contributions to the effective action. We have justified the assumption that the decay rate is much slower than the mass scale of the scalar field, by explicitly showing that the classical decay rate is exponentially suppressed. Moreover, we have first found that there are some critical values of charge (energy) at which the dominant decay channel vanishes accidentally. As a result, I-balls/oscillons stay at such critical points in most of their lifetime. In other words, I-balls/oscillons have approximate fixed points in their dynamics. We have ensured this essential nature of I-balls/oscillons by numerical simulations of the original relativistic 
scalar field theory. Our results indicate that the dynamical emergence of exponential separation of time scales leads to the approximately conserved quantity and the extraordinary longevity of the I-balls/oscillons.

While the main purpose of this paper is to clarify the classical longevity of I-balls/oscillons, it is desirable to include quantum effects if one would like to apply the I-balls/oscillons in cosmology. In our effective theory, the task is rather straightforward; that is, the inclusion of the loop diagrams. The real part of the loop contributions typically results in logarithmic corrections to the effective potential due to the renormalization running of coupling constants. The imaginary part of loop contributions has interesting effects. Since quantum fluctuations admit spontaneous relativistic particle production, the decay channels increase [52, 53]. For instance, the production of relativistic modes with

$p_{0} \simeq 2 \omega$ and $p \simeq \sqrt{4 \omega^{2}-m_{\phi}^{2}}$ can occur even in the $\mathbb{Z}_{2}$ symmetric case, which is otherwise prohibited as one can see from figure 5 . The quantum decay rate is suppressed by the smallness of $\epsilon \equiv\left(m_{\phi}-\omega\right) / m_{\phi}[46,52]$, while there is no exponential suppression since we do not need the gradients to hit the pole contrary to the classical decay [eq. (2.48)]. All one have to do is to include this term in the right-hand-side of eq. (2.48). Then we can estimate the lifetime of I-balls/oscillons including quantum effects.

Since we only assume that the configuration is dominated by the non-relativistic one, our formalism is rather generic. Therefore, it has a potential applicability to the formation of I-balls/oscillons, if one estimates the imaginary part of the effective action during the formation and could guarantee its smallness. This is a clear contrast to the $\epsilon$ expansion where one expands the field around the I-balls/oscillons solutions. We come back to this issue elsewhere. In addition, our formalism can also be used for rather generic condensates of non-relativistic scalar fields. For instance, it may be useful to study condensates of ultra light bosonic dark matter, such as fuzzy dark matter, axion stars, and so on [48-50, 62-77].

\section{Acknowledgments}

Feynman diagrams were drawn by means of [78]. This work is supported by Grant-inAid for Scientific Research from the Ministry of Education, Science, Sports, and Culture (MEXT), Japan, World Premier International Research Center Initiative (WPI Initiative), MEXT, Japan (K.M.), and JSPS Research Fellowships for Young Scientists (K.M., M.T., and M.Y.).

\section{A Interaction terms}

Here we summarize all the interaction terms between NR modes and the other modes for the sake of completeness. First, let us consider the case of

$$
V_{\mathrm{int}}=\frac{g_{4}}{4} \phi^{4}+\frac{g_{6}}{6} \phi^{6}
$$

Note here that the sign of $g_{4}$ is opposite to that in the main text. 
In this case, we have the following interaction terms. For $g_{4}$, we have

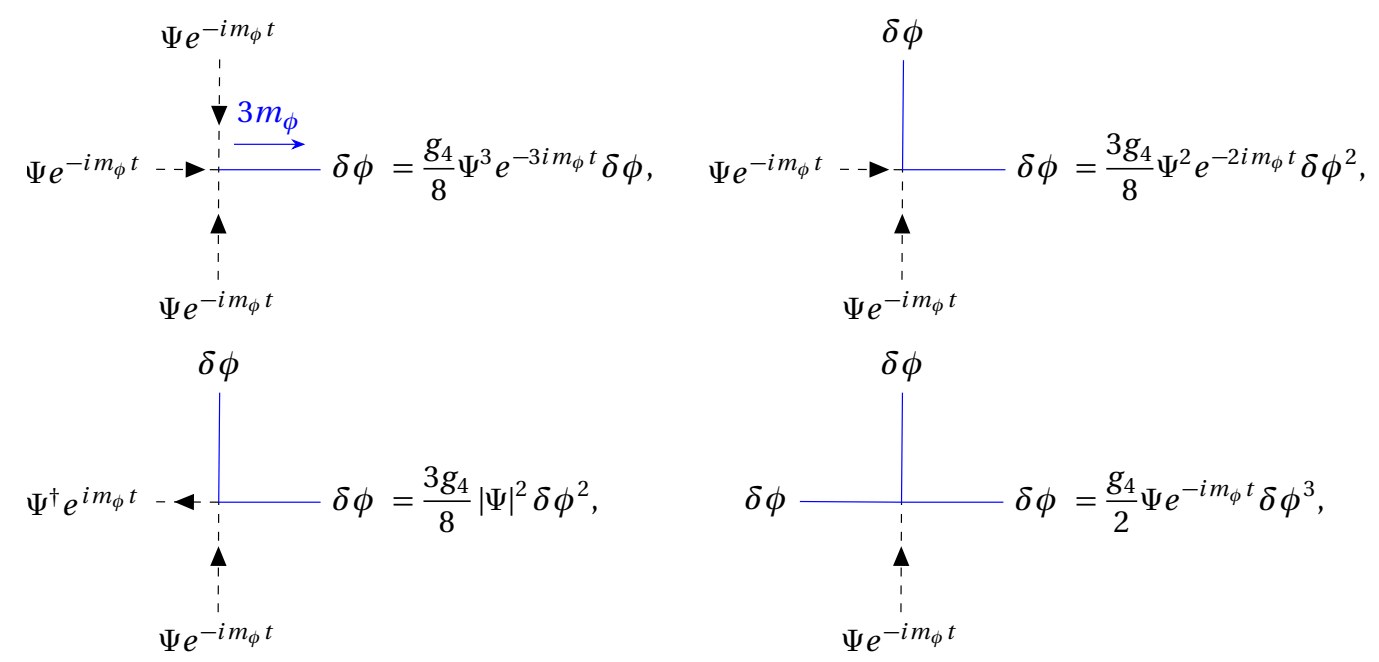

and those Hermite conjugates. For $g_{6}$, we have

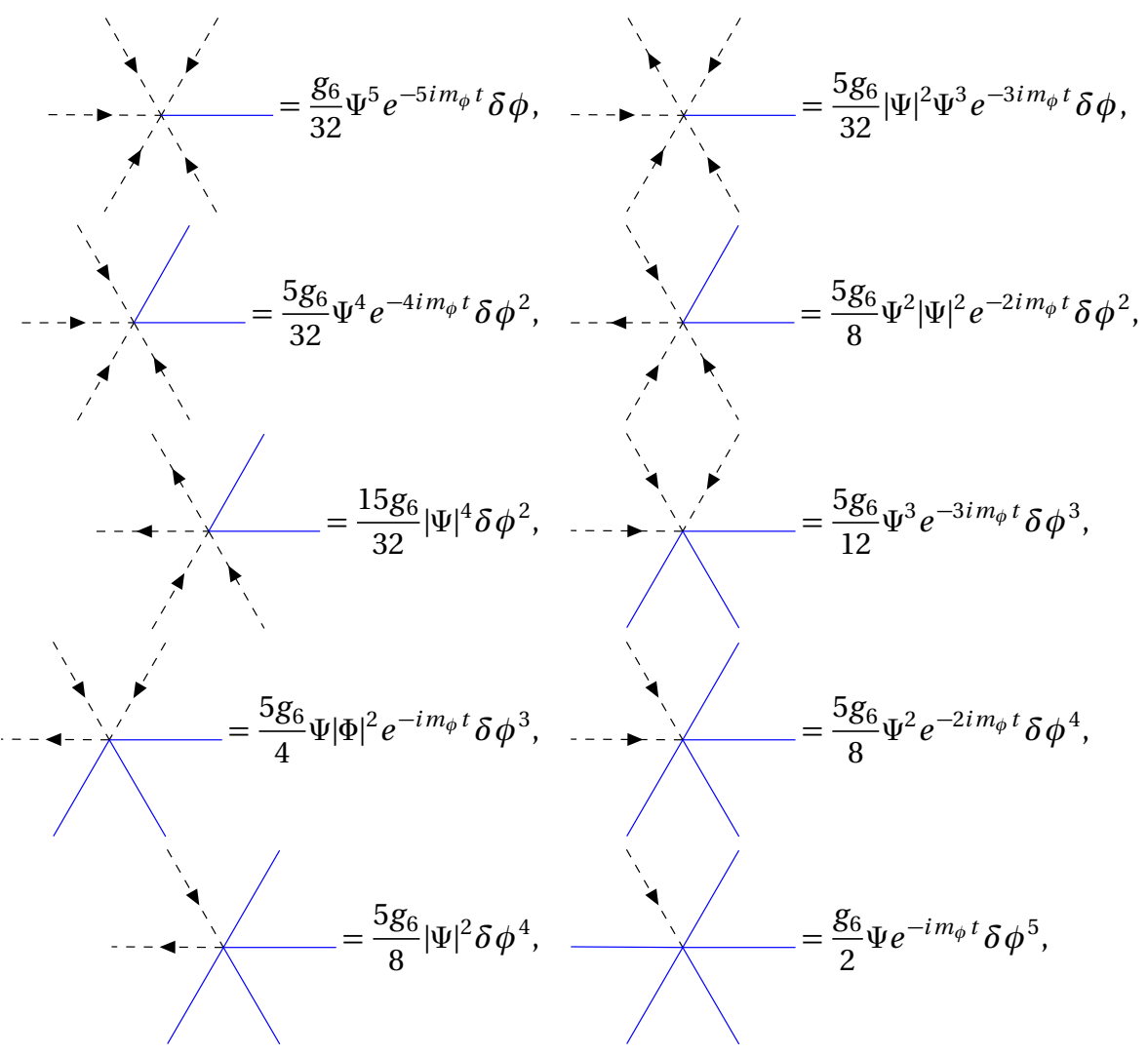

and those Hermite conjugates. Here we have omitted $\Phi$ and $\delta \phi$ in the legs of feynman diagrams for brevity.

As an example of $\mathbb{Z}_{2}$ breaking interaction, let us consider

$$
V_{\mathrm{int}}=\frac{g_{3}}{3} \phi^{3} .
$$


The interaction terms are the followings:

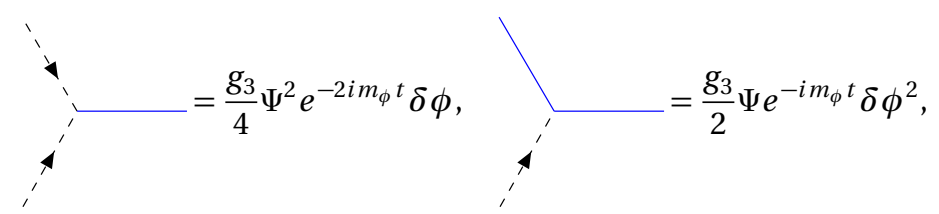

and those Hermite conjugates. We also have terms involving $\delta \phi_{V}$ in the case of $\mathbb{Z}_{2}$ breaking term:

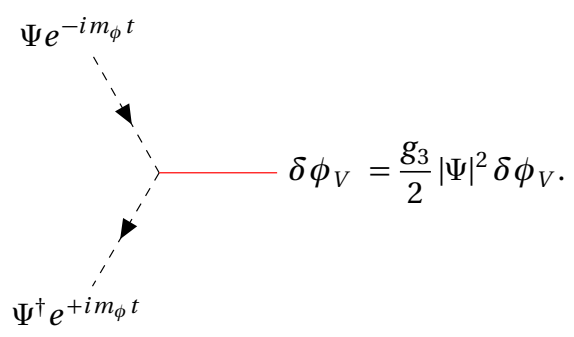

\section{B Higher order terms}

Here we summarize higher order terms in the effective action. Those terms are relevant if one applies our formalism to the double well potential. This is because the non-relativistic condition is marginally satisfied in this case, which calls for higher order terms in the non-relativistic expansion.

To be concrete, we start with a relativistic scalar field theory with the following potential:

$$
V_{\mathrm{int}}(\phi)=\frac{g_{3}}{3} \phi^{3}+\frac{g_{4}}{4} \phi^{4} .
$$

Here, note that there is a mass term in addition to this potential in our notation [see eq. (2.1)]. By performing the procedure described in section 2.1, we obtain the following effective potential for non-relativistic configurations:

$$
V_{\mathrm{eff}}(|\Psi|)=\sum_{n=2} c_{2 n}|\Psi|^{2 n}+f(|\Psi|, \nabla) .
$$

Note that the second term stands for corrections involving spatial gradients.

Up to the 8th dimensions, the coefficients are given by

$$
\begin{aligned}
c_{4}= & \frac{3 g_{4}}{8}-\frac{5 g_{3}^{2}}{12 m_{\phi}^{2}}+\frac{2\left(\mu / m_{\phi}\right) g_{3}^{2}}{9 m_{\phi}^{2}}, \\
c_{6}= & \frac{g_{4}^{2}}{128 m_{\phi}^{2}}+\frac{9\left(\mu / m_{\phi}\right) g_{4}^{2}}{512 m_{\phi}^{2}}+\frac{53 g_{4} g_{3}^{2}}{96 m_{\phi}^{4}}-\frac{55 g_{3}^{4}}{288 m_{\phi}^{6}}-\frac{421\left(\mu / m_{\phi}\right) g_{3}^{4}}{3456 m_{\phi}^{6}}, \\
c_{8}= & -\frac{8245 g_{3}^{6}}{62208 m_{\phi}^{10}}-\frac{3647\left(\mu / m_{\phi}\right) g_{3}^{6}}{124416 m_{\phi}^{10}}+\frac{3 g_{4}^{3}}{2048 m_{\phi}^{4}}+\frac{27\left(\mu / m_{\phi}\right) g_{4}^{3}}{4096 m_{\phi}^{4}} \\
& -\frac{737 g_{4}^{2} g_{3}^{2}}{1024 m_{\phi}^{6}}+\frac{13877\left(\mu / m_{\phi}\right) g_{3}^{2} g_{4}^{2}}{18432 m_{\phi}^{6}}+\frac{10775 g_{4} g_{3}^{4}}{13824 m_{\phi}^{8}}+\frac{3181\left(\mu / m_{\phi}\right) g_{3}^{4} g_{4}}{82944 m_{\phi}^{8}} .
\end{aligned}
$$


Here, we include the leading order correction coming from the difference between the "bare mass" $m_{\phi}$ and the "effective mass" $\omega\left(=m_{\phi}-\mu\right)$, which is much smaller than unity in most cases we are interested in. One can see that the expansion is good when $g_{3} \ll 1$ because of small coefficients. The terms associated with the coupling $g_{3}$ and having $\mathcal{O}(1)$ coefficients come from the integration of non-relativistic mode $\delta \phi_{V}$ [see eq. (2.12)]. Since $g_{3}=\mathcal{O}(1)$ in the case of double well potential, the higher order terms are relevant and the expansion may not be good unless $\phi \ll 1$. Still, we expect that the qualitative features can be extracted from the effective potential even if we truncate it at some order. We use it up to 8th orders in this paper.

Since I-balls/oscillons are non-relativistic objects, the spacial derivative is usually smaller than the oscillation time scale. This allows us to neglect higher-order derivative terms. Here, we write the function $f$ associated with the derivatives up to the second-order derivatives and the mass dimension 8 :

$$
\begin{aligned}
\left.f(|\Psi|, \nabla)\right|_{\text {mass dim } 6}= & \frac{g_{3}^{2}}{2 m_{\phi}^{4}}\left[\left(\nabla|\Psi|^{2}\right) \cdot\left(\nabla|\Psi|^{2}\right)+\frac{1}{18}\left(\nabla \Psi^{\dagger 2}\right) \cdot\left(\nabla \Psi^{2}\right)\right] \\
\left.f(|\Psi|, \nabla)\right|_{\text {mass dim } 8}= & \frac{g_{3}^{4}}{m_{\phi}^{8}}\left[\frac{1}{2304}\left(\nabla \Psi^{\dagger 3}\right) \cdot\left(\nabla \Psi^{3}\right)+\frac{7}{864}\left(|\Psi|^{2} \Psi^{2} \Delta \Psi^{\dagger 2}+|\Psi|^{2} \Psi^{\dagger 2} \Delta \Psi^{2}\right)\right. \\
& \left.\quad+\frac{19}{36}\left(\nabla|\Psi|^{4}\right) \cdot\left(\nabla|\Psi|^{2}\right)\right] \\
& +\frac{g_{3}^{2} g_{4}}{m_{\phi}^{6}}\left[\frac{1}{768}\left(\nabla \Psi^{\dagger 3}\right) \cdot\left(\nabla \Psi^{3}\right)+\frac{5}{192}\left(|\Psi|^{2} \Psi^{2} \Delta \Psi^{\dagger 2}+|\Psi|^{2} \Psi^{\dagger 2} \Delta \Psi^{2}\right)\right. \\
& \left.\quad-\frac{5}{4}\left(\nabla|\Psi|^{4}\right) \cdot\left(\nabla|\Psi|^{2}\right)\right] \\
& +\frac{g_{4}^{2}}{1024 m_{\phi}^{4}}\left(\nabla \Psi^{\dagger 3}\right) \cdot\left(\nabla \Psi^{3}\right) .
\end{aligned}
$$

We also write only relevant derivative terms with the mass dimension 10, which come from the integration of $\delta \phi_{V}$ :

$$
\begin{aligned}
\left.f(|\Psi|, \nabla)\right|_{\text {mass dim } 10 \text { from } \delta \phi_{V}}= & \frac{g_{3}^{6}}{m_{\phi}^{10}}\left[\frac{3539}{6912}\left(\nabla|\Psi|^{6}\right) \cdot\left(\nabla|\Psi|^{2}\right)+\frac{361}{2592}\left(\nabla|\Psi|^{4}\right) \cdot\left(\nabla|\Psi|^{4}\right)\right] \\
& +\frac{g_{3}^{4} g_{4}}{m_{\phi}^{8}}\left[-\frac{1879}{768}\left(\nabla|\Psi|^{6}\right) \cdot\left(\nabla|\Psi|^{2}\right)-\frac{95}{144}\left(\nabla|\Psi|^{4}\right) \cdot\left(\nabla|\Psi|^{4}\right)\right] \\
& +\frac{g_{3}^{2} g_{4}^{2}}{m_{\phi}^{6}}\left[\frac{1697}{1024}\left(\nabla|\Psi|^{6}\right) \cdot\left(\nabla|\Psi|^{2}\right)+\frac{25}{32}\left(\nabla|\Psi|^{4}\right) \cdot\left(\nabla|\Psi|^{4}\right)\right] .
\end{aligned}
$$

Open Access. This article is distributed under the terms of the Creative Commons Attribution License (CC-BY 4.0), which permits any use, distribution and reproduction in any medium, provided the original author(s) and source are credited.

\section{References}

[1] A.H. Guth, The Inflationary Universe: A Possible Solution to the Horizon and Flatness Problems, Phys. Rev. D 23 (1981) 347 [InSPIRE]. 
[2] A.D. Linde, A New Inflationary Universe Scenario: A Possible Solution of the Horizon, Flatness, Homogeneity, Isotropy and Primordial Monopole Problems, Phys. Lett. B 108 (1982) 389 [INSPIRE].

[3] Planck collaboration, P.A.R. Ade et al., Planck 2013 results. XVI. Cosmological parameters, Astron. Astrophys. 571 (2014) A16 [arXiv:1303.5076] [INSPIRE].

[4] K. Enqvist and M.S. Sloth, Adiabatic CMB perturbations in pre-big-bang string cosmology, Nucl. Phys. B 626 (2002) 395 [hep-ph/0109214] [INSPIRE].

[5] D.H. Lyth and D. Wands, Generating the curvature perturbation without an inflaton, Phys. Lett. B 524 (2002) 5 [hep-ph/0110002] [INSPIRE].

[6] T. Moroi and T. Takahashi, Effects of cosmological moduli fields on cosmic microwave background, Phys. Lett. B 522 (2001) 215 [Erratum ibid. B 539 (2002) 303] [hep-ph/0110096] [INSPIRE].

[7] R.D. Peccei and H.R. Quinn, CP Conservation in the Presence of Instantons, Phys. Rev. Lett. 38 (1977) 1440 [INSPIRE].

[8] S. Weinberg, A New Light Boson?, Phys. Rev. Lett. 40 (1978) 223 [InSPIRE].

[9] J. Preskill, M.B. Wise and F. Wilczek, Cosmology of the Invisible Axion, Phys. Lett. B 120 (1983) 127 [INSPIRE].

[10] L.F. Abbott and P. Sikivie, A Cosmological Bound on the Invisible Axion, Phys. Lett. B 120 (1983) 133 [INSPIRE].

[11] M. Dine and W. Fischler, The Not So Harmless Axion, Phys. Lett. B 120 (1983) 137 [INSPIRE].

[12] I. Affleck and M. Dine, A New Mechanism for Baryogenesis, Nucl. Phys. B 249 (1985) 361 [INSPIRE].

[13] H. Murayama and T. Yanagida, Leptogenesis in supersymmetric standard model with right-handed neutrino, Phys. Lett. B 322 (1994) 349 [hep-ph/9310297] [INSPIRE].

[14] M. Dine, L. Randall and S.D. Thomas, Baryogenesis from flat directions of the supersymmetric standard model, Nucl. Phys. B 458 (1996) 291 [hep-ph/9507453] [INSPIRE].

[15] Ya.B. Zeldovich, I.Yu. Kobzarev and L.B. Okun, Cosmological Consequences of the Spontaneous Breakdown of Discrete Symmetry, Zh. Eksp. Teor. Fiz. 67 (1974) 3 [INSPIRE].

[16] P. Sikivie, Of Axions, Domain Walls and the Early Universe, Phys. Rev. Lett. 48 (1982) 1156 [INSPIRE].

[17] A. Vilenkin and A.E. Everett, Cosmic Strings and Domain Walls in Models with Goldstone and PseudoGoldstone Bosons, Phys. Rev. Lett. 48 (1982) 1867 [INSPIRE].

[18] S.R. Coleman, Q Balls, Nucl. Phys. B 262 (1985) 263 [Erratum ibid. B 269 (1986) 744] [INSPIRE].

[19] A. Kusenko and M.E. Shaposhnikov, Supersymmetric $Q$ balls as dark matter, Phys. Lett. B 418 (1998) 46 [hep-ph/9709492] [INSPIRE].

[20] K. Enqvist and J. McDonald, Q balls and baryogenesis in the MSSM, Phys. Lett. B 425 (1998) 309 [hep-ph/9711514] [inSPIRE].

[21] K. Enqvist and J. McDonald, B-ball baryogenesis and the baryon to dark matter ratio, Nucl. Phys. B 538 (1999) 321 [hep-ph/9803380] [INSPIRE]. 
[22] S. Kasuya and M. Kawasaki, Q ball formation through Affleck-Dine mechanism, Phys. Rev. D 61 (2000) 041301 [hep-ph/9909509] [INSPIRE].

[23] S. Kasuya and M. Kawasaki, Q ball formation in the gravity mediated SUSY breaking scenario, Phys. Rev. D 62 (2000) 023512 [hep-ph/0002285] [INSPIRE].

[24] S. Kasuya and M. Kawasaki, Q ball formation: Obstacle to Affleck-Dine baryogenesis in the gauge mediated SUSY breaking?, Phys. Rev. D 64 (2001) 123515 [hep-ph/0106119] [INSPIRE].

[25] I.L. Bogolyubsky and V.G. Makhankov, On the Pulsed Soliton Lifetime in Two Classical Relativistic Theory Models, JETP Lett. 24 (1976) 12 [INSPIRE].

[26] H. Segur and M.D. Kruskal, Nonexistence of Small Amplitude Breather Solutions in $\phi^{4}$ Theory, Phys. Rev. Lett. 58 (1987) 747 [inSPIRE].

[27] M. Gleiser, Pseudostable bubbles, Phys. Rev. D 49 (1994) 2978 [hep-ph/9308279] [INSPIRE].

[28] E.J. Copeland, M. Gleiser and H.R. Muller, Oscillons: resonant configurations during bubble collapse, Phys. Rev. D 52 (1995) 1920 [hep-ph/9503217] [INSPIRE].

[29] M. Gleiser and A. Sornborger, Longlived localized field configurations in small lattices: application to oscillons, Phys. Rev. E 62 (2000) 1368 [patt-sol/9909002] [INSPIRE].

[30] E.P. Honda and M.W. Choptuik, Fine structure of oscillons in the spherically symmetric phi**4 Klein-Gordon model, Phys. Rev. D 65 (2002) 084037 [hep-ph/0110065] [INSPIRE].

[31] S. Kasuya, M. Kawasaki and F. Takahashi, I-balls, Phys. Lett. B 559 (2003) 99 [hep-ph/0209358] [INSPIRE].

[32] M. Gleiser, d-dimensional oscillating scalar field lumps and the dimensionality of space, Phys. Lett. B 600 (2004) 126 [hep-th/0408221] [INSPIRE].

[33] G. Fodor, P. Forgacs, P. Grandclement and I. Racz, Oscillons and Quasi-breathers in the $\phi^{4}$ Klein-Gordon model, Phys. Rev. D 74 (2006) 124003 [hep-th/0609023] [inSPIRE].

[34] M. Hindmarsh and P. Salmi, Numerical investigations of oscillons in 2 dimensions, Phys. Rev. D 74 (2006) 105005 [hep-th/0606016] [INSPIRE].

[35] P.M. Saffin and A. Tranberg, Oscillons and quasi-breathers in D+1 dimensions, JHEP 01 (2007) 030 [hep-th/0610191] [INSPIRE].

[36] G. Fodor, P. Forgacs, Z. Horvath and M. Mezei, Computation of the radiation amplitude of oscillons, Phys. Rev. D 79 (2009) 065002 [arXiv:0812.1919] [InSPIRE].

[37] M. Gleiser and D. Sicilia, Analytical Characterization of Oscillon Energy and Lifetime, Phys. Rev. Lett. 101 (2008) 011602 [arXiv:0804.0791] [INSPIRE].

[38] G. Fodor, P. Forgacs, Z. Horvath and M. Mezei, Radiation of scalar oscillons in 2 and 3 dimensions, Phys. Lett. B 674 (2009) 319 [arXiv:0903.0953] [INSPIRE].

[39] M. Gleiser and D. Sicilia, A General Theory of Oscillon Dynamics, Phys. Rev. D 80 (2009) 125037 [arXiv: 0910 . 5922] [INSPIRE].

[40] M.A. Amin and D. Shirokoff, Flat-top oscillons in an expanding universe, Phys. Rev. D 81 (2010) 085045 [arXiv: 1002.3380] [INSPIRE].

[41] M.A. Amin, R. Easther, H. Finkel, R. Flauger and M.P. Hertzberg, Oscillons After Inflation, Phys. Rev. Lett. 108 (2012) 241302 [arXiv:1106.3335] [INSPIRE]. 
[42] P. Salmi and M. Hindmarsh, Radiation and Relaxation of Oscillons, Phys. Rev. D 85 (2012) 085033 [arXiv: 1201.1934 ] [INSPIRE].

[43] E.A. Andersen and A. Tranberg, Four results on $\phi^{4}$ oscillons in D+1 dimensions, JHEP 12 (2012) 016 [arXiv:1210.2227] [INSPIRE].

[44] K.D. Lozanov and M.A. Amin, End of inflation, oscillons and matter-antimatter asymmetry, Phys. Rev. D 90 (2014) 083528 [arXiv:1408.1811] [InSPIRE].

[45] P.M. Saffin, P. Tognarelli and A. Tranberg, Oscillon Lifetime in the Presence of Quantum Fluctuations, JHEP 08 (2014) 125 [arXiv: 1401.6168] [INSPIRE].

[46] K. Mukaida and M. Takimoto, Correspondence of I- and Q-balls as Non-relativistic Condensates, JCAP 08 (2014) 051 [arXiv: 1405.3233] [INSPIRE].

[47] M. Kawasaki, F. Takahashi and N. Takeda, Adiabatic Invariance of Oscillons/I-balls, Phys. Rev. D 92 (2015) 105024 [arXiv:1508.01028] [INSPIRE].

[48] J. Berges and J. Jaeckel, Far from equilibrium dynamics of Bose-Einstein condensation for Axion Dark Matter, Phys. Rev. D 91 (2015) 025020 [arXiv:1402.4776] [INSPIRE].

[49] S. Davidson, Axions: Bose Einstein Condensate or Classical Field?, Astropart. Phys. 65 (2015) 101 [arXiv:1405.1139] [INSPIRE].

[50] E. Braaten, A. Mohapatra and H. Zhang, Nonrelativistic Effective Field Theory for Axions, Phys. Rev. D 94 (2016) 076004 [arXiv:1604.00669] [InSPIRE].

[51] G.D. Moore, Condensates in Relativistic Scalar Theories, Phys. Rev. D 93 (2016) 065043 [arXiv: 1511.00697] [INSPIRE].

[52] M.P. Hertzberg, Quantum Radiation of Oscillons, Phys. Rev. D 82 (2010) 045022 [arXiv: 1003.3459] [INSPIRE].

[53] M. Kawasaki and M. Yamada, Decay rates of Gaussian-type-I-balls and Bose-enhancement effects in 3+1 dimensions, JCAP 02 (2014) 001 [arXiv: 1311.0985] [INSPIRE].

[54] P.M. Saffin, Recrudescence of massive fermion production by oscillons, arXiv: 1612.02014 [INSPIRE].

[55] A. Kusenko, Small Q balls, Phys. Lett. B 404 (1997) 285 [hep-th/9704073] [InSPIRE].

[56] K. Enqvist and M. Laine, Q-ball dynamics from atomic Bose-Einstein condensates, JCAP 08 (2003) 003 [cond-mat/0304355] [INSPIRE].

[57] S.R. Coleman, V. Glaser and A. Martin, Action Minima Among Solutions to a Class of Euclidean Scalar Field Equations, Commun. Math. Phys. 58 (1978) 211 [inSPIRE].

[58] I.E. Gulamov, E. Ya. Nugaev and M.N. Smolyakov, Theory of U(1) gauged Q-balls revisited, Phys. Rev. D 89 (2014) 085006 [arXiv:1311.0325] [INSPIRE].

[59] R. Friedberg, T.D. Lee and A. Sirlin, A Class of Scalar-Field Soliton Solutions in Three Space Dimensions, Phys. Rev. D 13 (1976) 2739 [InSPIRE].

[60] T.D. Lee and Y. Pang, Nontopological solitons, Phys. Rept. 221 (1992) 251 [inSPIRE].

[61] M.I. Tsumagari, The Physics of Q-Balls, PhD thesis, Nottingham University, Nottingham U.K. (2009), arXiv:0910.3845 [INSPIRE].

[62] J. Eby, P. Suranyi and L.C.R. Wijewardhana, The Lifetime of Axion Stars, Mod. Phys. Lett. A 31 (2016) 1650090 [arXiv:1512.01709] [INSPIRE]. 
[63] R. Ruffini and S. Bonazzola, Systems of selfgravitating particles in general relativity and the concept of an equation of state, Phys. Rev. 187 (1969) 1767 [INSPIRE].

[64] C.J. Hogan and M.J. Rees, Axion miniclusters, Phys. Lett. B 205 (1988) 228 [INSPIRE].

[65] E.W. Kolb and I.I. Tkachev, Axion miniclusters and Bose stars, Phys. Rev. Lett. 71 (1993) 3051 [hep-ph/9303313] [INSPIRE].

[66] E. Seidel and W.-M. Suen, Formation of solitonic stars through gravitational cooling, Phys. Rev. Lett. 72 (1994) 2516 [gr-qc/9309015] [INSPIRE].

[67] W. Hu, R. Barkana and A. Gruzinov, Cold and fuzzy dark matter, Phys. Rev. Lett. 85 (2000) 1158 [astro-ph/0003365] [INSPIRE].

[68] F.S. Guzman and L.A. Urena-Lopez, Gravitational cooling of self-gravitating Bose-Condensates, Astrophys. J. 645 (2006) 814 [astro-ph/0603613] [INSPIRE].

[69] P. Sikivie and Q. Yang, Bose-Einstein Condensation of Dark Matter Axions, Phys. Rev. Lett. 103 (2009) 111301 [arXiv:0901.1106] [INSPIRE].

[70] J. Barranco and A. Bernal, Self-gravitating system made of axions, Phys. Rev. D 83 (2011) 043525 [arXiv: 1001.1769] [INSPIRE].

[71] O. Erken, P. Sikivie, H. Tam and Q. Yang, Cosmic axion thermalization, Phys. Rev. D 85 (2012) 063520 [arXiv:1111.1157] [INSPIRE].

[72] P.H. Chavanis and L. Delfini, Mass-radius relation of Newtonian self-gravitating Bose-Einstein condensates with short-range interactions: II. Numerical results, Phys. Rev. D 84 (2011) 043532 [arXiv:1103.2054] [INSPIRE].

[73] J. Eby, P. Suranyi, C. Vaz and L.C.R. Wijewardhana, Axion Stars in the Infrared Limit, JHEP 03 (2015) 080 [Erratum ibid. 1611 (2016) 134] [arXiv: 1412.3430] [INSPIRE].

[74] A.H. Guth, M.P. Hertzberg and C. Prescod-WEinstein, Do Dark Matter Axions Form a Condensate with Long-Range Correlation?, Phys. Rev. D 92 (2015) 103513 [arXiv: 1412.5930] [INSPIRE].

[75] E. Braaten, A. Mohapatra and H. Zhang, Dense Axion Stars, Phys. Rev. Lett. 117 (2016) 121801 [arXiv: 1512.00108] [INSPIRE].

[76] L. Hui, J.P. Ostriker, S. Tremaine and E. Witten, Ultralight scalars as cosmological dark matter, Phys. Rev. D 95 (2017) 043541 [arXiv: 1610.08297] [INSPIRE].

[77] J. Eby, M. Leembruggen, P. Suranyi and L.C.R. Wijewardhana, Collapse of Axion Stars, JHEP 12 (2016) 066 [arXiv: 1608.06911] [INSPIRE].

[78] J. Ellis, TikZ-Feynman: Feynman diagrams with TikZ, Comput. Phys. Commun. 210 (2017) 103 [arXiv: 1601.05437 ] [INSPIRE]. 